\title{
1 Molecular dynamics-driven drug discovery: 2 Leaping forward with confidence
}

3

4

5

6 7

16 *Corresponding author

17 Phone: 1-780-492-5783

18 Email: kbarakat@ualberta.ca

19 Canada.

\section{8}

\section{Aravindhan Ganesan ${ }^{1}$, Michelle L. Coote ${ }^{2}$ and Khaled Barakat ${ }^{1 *}$}

${ }^{1}$ Faculty of Pharmacy and Pharmaceutical Sciences, University of Alberta, Edmonton, Alberta,

${ }^{2}$ ARC Centre of Excellence for Electromaterials Science, Research School of Chemistry, Australian National University, Canberra ACT 2601, Australia.

[Keywords: Molecular dynamics, drug discovery, computer-aided drug design, protein flexibility, binding free energy]

(21-September-2016) 


\section{Abstract}

2 Given the high costs and time in developing a commercial drug, it remains important to 3 constantly reform the drug discovery pipeline with novel technologies that can narrow 4 down on the most promising lead compounds for clinical testing. The past decade has

5 witnessed tremendous growth in computational capabilities that allow in silico approaches

6 to expedite drug discovery processes. Molecular dynamics (MD) has become a particularly 7 important tool in drug design and discovery. From classical MD methods to more 8 sophisticated hybrid classical/quantum mechanical approaches, MD simulations are now 9 able to offer extraordinary insights into ligand-receptor interactions. In this review, we 10 discuss how the applications of MD approaches are significantly transforming the current 11 drug discovery and development efforts.

12

13 


\section{Introduction}

2 The quest for new drugs has always remained crucial throughout human history. From

3 epidemic influenza of 1800s and 1900s[1] to the very recent Ebola virus outbreaks[2], the

4 world's population has constantly faced several dreadful epidemics, in addition to life-

5 threatening diseases such as cancers. Thus drug discovery continues to be the most

6 significant challenge for the scientific community. The overall drug discovery process,

7 from the identification of potential lead compounds to the FDA approval of a drug, is not

8 only extremely complex but also highly expensive and time consuming. A very recent

9 report $^{1}$ published by the Tufts Center for the Study of Drug Development (CSDD)

10 estimates the overall costs for developing an approved drug at a staggering \$2.6 billion,

11 with an average of approximately 14 years to complete the entire development cycle of a

12 single drug (from research labs to market)[3].

14 Drug design and development have matured over the last two decades by exploiting the 15 advantages of new experimental techniques and complementary technologies. The early 16 1990s saw rapid advancements in combinatorial chemistry and high-throughput gene

17 sequencing technology. These allowed the synthesis of huge compound libraries within a

18 short span of time and their screening for various targets, thereby accelerating the discovery

19 processes. This raised the hope of transforming the drug discovery field, making the natural

20 products obsolete. But over time, the field of combinatorial chemistry started to face a lot

21 of technical challenges. Particularly, the combinatorial libraries did not cover many

22 structurally diverse compounds[4]. Further, the compounds in these libraries were also not

23 stereochemically rich as the natural products. Therefore, the steep expansion in these

24 compound libraries did not provide the expected fruitful outcomes; on the contrary, they

25 only escalated the costs of testing[5] and resulted in reduced success rates. For example,

26 until recently, only two compounds generated de novo have reacted the market as a drug[6].

27 One of them is sorafenib from Bayer, which was first approved by FDA in 2005 as a drug

28 for cancer. The second drug that was possibly generated from de novo design is ataluren,

29 which was approved in the European union in 2014 as a drug for the treatment of genetic

30 disorders[6]. Nevertheless, there have been some significant efforts towards improving the

${ }^{1}$ http://csdd.tufts.edu/news/complete story/pr tufts csdd 2014 cost study 
1 combinatorial chemistry field. For instance, some of the schemes for addressing the lack

2 of diversity were developed; and this includes diversity-oriented synthesis[7], which

3 employs a "build/couple/pair strategy"[8]. In addition, strategies, such as 'split and pool

4 solid phase synthesis', were developed as more powerful approach for synthesizing huge

5 combinatorial chemistry. Despite many efforts, the field of combinatorial chemistry has

6 still not reached its full capacity. Kodadek[9] discusses various recent advances in the

7 combinatorial chemistry. This has led to a focus on computational methods as low-cost

8 tools for driving the early search process for compounds with desired biological activity

9 and pharmacological profiles, before initiating experiments.

11 Structure-based drug design (SBDD) is one of the vital computational approaches that has

12 been found to be very effective in the identification of hits for in-vitro testing. As the name

13 indicates, in principle, knowledge of the three-dimensional structures of proteins and the

14 ligands are mandatory to perform SBDD. Recently, there has been dramatic accumulation

15 of biological data, from gene sequences to three-dimensional structures of proteins and

16 compound databases, which offers excellent support to SBDD research. As of June 2016,

17 the Protein Data Bank (PDB) (www.pdb.org) contains more than one hundred thousand

18 experimentally-determined (e.g., via X-ray, NMR and electron microscopy) protein

19 structures, of which almost $26 \%$ correspond to human proteins. The UniProtKB/Swiss-Prot

20 genome database (www.uniprot.org) contains $\sim 540,000$ amino acid sequences. These huge

21 databases offer a gamut of potential targets for several human diseases. Moreover, when

22 the experimentally-determined 3D structures of any proteins (or enzymes) are not available

23 in the PDB, computational models of the unknown proteins for subsequent in-silico studies

24 can be constructed using SBDD-based methods such as homology modelling, threading

25 and de novo designing[10]. The success of virtual screening (see Glossary) and SBDD is

26 also dependent on the availability of different compound libraries that comprise chemical

27 compounds from diverse structural classes, so as to increase the probability of obtaining

28 novel hits. There are a number of freely available compound databases, such as

29 ZINC15[11,12] ( $\sim 120$ million compounds), Chemspider[13] (35 million compounds),

30 ChEMBL[14] ( 2 million compounds), DrugBank[15] ( 14000 compounds),

31 PubChem[16] (64 million compounds), among others. 
2 When a specific target and compound libraries are selected, molecular docking-based

3 virtual high-throughput screening is employed to identify only those compounds (from the

4 libraries) with higher affinities to the protein's active site[17]. The proteins are dynamic

5 biological molecules and their flexibilities play vital roles in the process of ligand

6 recognition, and thus in SBDD. In addition, ligand binding also tends to induce measurable

7 levels of conformational changes in the proteins so as to adapt a biophysical state that is

8 suitable to form a strongly-bound complex (known as induced-fit effects). Nevertheless,

9 accounting for receptor flexibilities remains a major challenge and regular molecular

10 docking methods are mostly unable to capture such conformational changes in proteins.

12 Molecular dynamics (MD) is a computational method that can take on this challenge and

13 predict the time-dependent behaviour of a molecular system, thus becoming an invaluable

14 tool in SBDD. It has been particularly valuable in exploring the energy landscapes of

15 proteins and identifying their physiological conformations, which are, in many cases, not

16 even accessible through high-resolution experimental techniques. MD is also useful in the

17 structural refinements of post-docking complexes, such that the complementarity between

18 the ligand and the receptor are enhanced in the complex-state, thereby allowing better re-

19 scoring of complexes.

21 This review will discuss in detail the various applications of MD approaches in modern

22 drug discovery efforts. Although, there have been a number of recent reviews[18-26]

23 focussing on the usefulness of MD in drug design, they are mostly focussed on the

24 theoretical background, applications of MD for accounting protein flexibility and selected

25 few binding free energy methods. However, the current review aims to complement the

26 existing ones in the literature, by addressing various aspects of SBDD, for which MD

27 methods and QM/MM approaches can offer some valuable solutions. The review begins

28 by briefly introducing molecular docking and virtual screening in SBDD. We discuss the

29 recent developments in docking methods and how they struggle to account protein

30 flexibility in SBDD. Subsequently, we discuss in detail how MD is helping to fill this gap

31 and various applications of MD in SBDD, including post-docking structural refinements 
1 and accurate binding free energy estimations. Various binding free energy methods and

2 their recent developments are presented, along with a number of examples. In addition, we

3 also discuss an emerging trend of using solvent information more explicitly from MD

4 simulations, which provide significant information the effects of water molecules in drug

5 design. Further, we also caution about various limitations in MD methods and,

6 subsequently, we discuss about the applications of advanced hybrid QM/MM MD in drug

7 design. Finally, we present our perspectives by presenting a simple and practical workflow

8 for integrating various computational methods discussed in this review for SBDD.

10 Molecular docking and flexibility challenges

11 Molecular docking (see Glossary) protocols predict the optimal placement of each of the 12 compounds within a pre-defined active site of a protein target. They generate a 13 comprehensive set of conformations of the ligand-receptor complex (predominantly based

14 on the ligand poses). These poses are subsequently ranked based on their stability using 15 different scoring functions[27]. There are a number of programs for ligand-protein 16 docking, including, DOCK[28], AutoDock[29], Gold[30], and GLIDE[31]. These

17 docking-based methods have been of great use in modern drug discovery campaigns, 18 mainly because of their speed and simple set-ups.

20 Early docking methods assumed that the ligand-protein binding phenomenon could be 21 modelled as a simple 'lock-and-key' scheme. That is, the aim was to identify a ligand (i.e.,

22 a key) with the exact shape complementarity to fit within a stiff active site cavity (as a 23 keyhole) of the protein. In this way, most early docking algorithms treated the ligand and 24 the receptor as two rigid counterparts. This assumption holds well only for very rare cases, 25 such as the trypsin-BPTI complex, in which the interface of the bound and unbound states 26 is almost identical in their conformations[32]. However, it does not reflect the reality in 27 vast majority of cases, where both ligands and receptors undergo mutual changes to 28 accommodate each other in the complex state. Thus, ligand-protein binding mechanism is 29 now described as a 'hand-and-glove' scheme (Fig. 1), indicating that the best fit is still an 30 essential factor but under a flexible environment[33]. Most of the current docking software 31 programs have adopted ligand sampling as one of the basic elements in their docking 
1 protocols. Several sampling algorithms, such as shape matching, systematic search and 2 stochastic algorithms, are currently employed in docking to generate several ligand 3 conformers (often referred as poses) around the given receptor environment[34]. For 4 example, software programs such as GLIDE[31] and LUDI[35] implement systematic 5 search methods in docking; while AutoDock incorporates stochastic methods for

6 accounting ligand flexibility in docking. Thus, there have been significant advancements 7 in the methods to allow exhaustive ligand flexibility in docking-based virtual 8 screening[34].

10 On the other hand, protein flexibility has been almost ignored in docking calculations. Very

11 few techniques, such as soft-docking and rotamer libraries[34,36], have been developed to 12 tackle this problem. In soft-docking, the protein flexibility is implicitly included during the 13 calculation, by softening the interatomic van der Waals terms in the scoring function such 14 that it allows small levels of overlaps between the receptor and ligand[34,36]. Software 15 programs such as GOLD and AutoDock implements soft-docking. Some programs attempt 16 to implement protein conformational changes into docking calculations by treating the side 17 chains as flexible, while retaining the rigidity of backbone atoms $[34,36]$. These methods

18 employ rotamer libraries, which comprise a list of side-chain conformations determined by 19 experiments and statistical analyses. GLIDE[31], for example, adapts an induced-fit

20 docking method, where selected side chains are mutated into alanine residues to avert steric 21 clashes during docking[31]. Later on, these side-chains' conformations are adjusted to 22 generate possible configurations that can adopt to the new environment, followed by an 23 energy minimization of the binding site.

25 Nevertheless, such attempts only allow local movements of some selected residues in the 26 active site, but are not able to capture the overall effects of ligand binding on the 27 conformation of proteins. To overcome that, an ensemble of protein structures can be used 28 to account for the full receptor flexibility during docking. This method has become one of 29 the most widely accepted techniques in SBDD. In this approach, all protein structures are 30 combined together to form a single representation [18,36], so that it can include 31 conformational changes occurring during the ligand binding process. This is usually 
1 achieved by averaging the grids of the different protein conformations (in the ensemble)

2 into a single global receptor grid and employing this final grid in molecular docking.

3 Knegtel et al[37] made one of early attempts in employing an averaged grid that is

4 generated from different experimentally-determined structures for ligand docking. The

5 authors employed this approach for different test cases, including HIV protease, ras p21

6 protein, uteroglobins and retinol binding protein. They found that the averaged grids

7 approach exhibited better accuracies when compared to those of a single structure. The

8 issue of protein flexibility in docking was also addressed by using a united description

9 scheme[38]. In this way, multiple experimentally-derived protein structures are

10 superimposed, where the similar segments in the ensemble structures are aligned and fused

11 together, while the variable regions are used as an ensemble. The ensemble of varied

12 segments of proteins is combinatorially-explored to produce possible new conformations

13 of proteins for docking calculations $[18,34]$. However, this approach relied heavily on the

14 quality of the ligand conformational sampling. In addition, such approaches account only

15 for the ligand-protein interaction energy where the internal energy of the protein is mostly

16 neglected[18] (Fig. 2).

18 An alternate ensemble-based strategy, to model protein flexibility in molecular docking, is

19 to explicitly consider multiple individual receptor conformations[39] and perform rigid

20 docking of ligands against all those target structures. An ensemble of protein configurations

21 is usually generated from an NMR structure of the chosen receptor or a set of X-ray crystal

22 structures for the same receptor but with different ligands. Nevertheless, the main pitfall

23 with using an ensemble of X-ray crystal structures is that the subsequent docking (or virtual

24 screening) could be biased towards the structures available. This could be even more

25 troublesome if all the available structures are co-crystalized with analogous ligands. On the

26 other hand, in the absence of those experimental structures, modeling and MD simulations

27 can be carried out to collect statistically significant protein conformations from the

28 resulting (MD) trajectories. More discussions about this strategy are provided in the

29 following sections. In fact, this combination-approach (i.e., mixing MD and molecular

30 docking) is becoming more common[40-43], irrespective of the availability of

31 experimental structures. For example, in a recent study, Campell et al[44] presented an 
1 approach that employs a biased-MD simulation on the known X-ray crystal structure(s) of

2 ligand-protein complex(es), followed by rigid ligand docking to identify the best ranking

3 pose for the complex(es). To demonstrate this scheme, the authors selected two test

4 systems, cyclin-dependent kinase 2 (CDK2) and factor Xa (FXa) [44]. The authors

5 collected the available crystal structures of these systems and performed MD simulations

6 by introducing an external bias potential to retain the initial ligand conformation, thereby

7 also maintaining the binding state that has been known to exist. Later, the authors collected

8 a cluster of protein conformations from the MD trajectory and employed them for the

9 ensemble-based docking of a new set of ligands in the known pocket. This work[44]

10 demonstrates that despite the availability of crystal structures, MD simulations may be very

11 useful to account protein flexibility in docking-based virtual screening. A recent study[45]

12 showed that enrichment performances of virtual screening against three different targets,

13 neuraminidase, HIV protease and peroxisome proliferator-activated alpha receptors,

14 displayed excellent improvements when employing MD-based screening. Therefore, MD

15 methods are now recognized as a valuable tool in SBDD.

\section{Classical molecular dynamics}

18 Molecular dynamics (MD) is the most widely employed computational technique to study

19 the equilibration structures and dynamic interactions of biological systems [20,24,40-43].

20 They extend insights into time-dependent variations and configurational changes in the

21 structures of the biological systems, which may be related to their functionalities[46].

22 Classical molecular dynamics regards atoms as solid spheres and the bonds connecting 23 them as springs. This allows the atoms in the system to only oscillate within a specified

24 distance. Classical MD is based on Newton's equations of motion,

$$
m_{i} \frac{\delta^{2} r_{i}}{\delta t^{2}}=F_{i}
$$

26 Here, $F_{i}$ is the component of the net force acting on the i-th atom with a mass, $m_{i} . r_{i}$

27 denotes the position of the atom at time t. The force can then be computed as, 


$$
F_{i}=-\frac{\delta U\left(r_{1}, r_{2}, \ldots r_{n}\right)}{\delta r_{i}}
$$

2 where, $U\left(r_{1}, r_{2}, \ldots r_{n}\right)$ is the potential energy function of the specific conformation and can

3 be described by using the concept of a force field with pre-defined parameters [47]. A

4 force field is a mathematical expression comprising the functional form of the potential

5 energy, which includes the possible bonded (bonds, angles and dihedrals) and non-bonded

6 interaction (van der Waals potentials and Coulomb potentials) terms between the different

7 atoms in the system. The bond stretching and angle terms are commonly modelled using a

8 harmonic potential function, while the dihedrals are expressed as a cosine function. The

9 non-bonded terms are modelled using Lennard-Jones potentials[48] and Coulomb's law.

10 Particle-mesh Ewald (PME) method[49] under periodic boundary conditions is normally

11 employed in the classical MD simulations in order to treat long-range electrostatic

12 interactions in the system. A number of force fields have been developed for MD

13 simulations of biological systems, such as CHARMM[50], AMBER[51], GROMOS[52],

14 etc. Most of these methods have different functional forms to treat MD simulations, which

15 makes it difficult to transfer parameters from one force field to another.

17 It is generally difficult to compare the performance of different force fields, as the outputs

18 will significantly depend on the type of system and properties studied[53]. However, there

19 have been some efforts to compare different force fields and most of them find that the

20 results concerning the structure and dynamics of systems could vary depending on the force

21 field. For example, Todorova et al[54] compared five popular force fields, such as

22 CHARMM27, OPLS, AMBER03, and the united-atom GROMOS 43A1 and GROMOS

23 53A6 force fields, for simulating insulin. The study addressed the effects of each force field

24 on the conformational evolution and structural properties of the peptides and compared

25 them against the established experimental data. The results found that different structural

26 trends emerged depending on the force field used; however, the CHARMM27 and

27 GROMOS 43A1 delivered the best representation of the experimental behavior[54].

28 Similar conclusions were drawn from a number of other studies as well; but some studies

29 concluded that no major differences (in properties and performance) were detected when 
1 comparing different force fields. Therefore, it is important to make a careful selection of a

2 force field before employing it in MD simulations. 'Learning from experience' is one of

3 the practical approaches for choosing a force field for MD simulations. Before choosing a

4 force field, the users need to be clear about the system they are working on and what is the

5 key question (or property) that they are trying to address through MD simulations.

6 Subsequently, the users need to do literature search to find out if MD simulations of similar

7 systems or properties have been reported earlier and if yes, what types of force fields were

8 applied to those simulations. If more than one force field have been applied, then which

9 one among them was able to provide more accurate results needs to be identified. It is also

10 important to benchmark the selected set of atomic force fields to test against some reliable

11 metrics. Sometimes the choice of force field may also depend on the type of water models

12 involved in the simulations, as force fields have been developed for certain water models

13 (such as TIP3P, TIP4 and SPC) [54,55]. For instance, it has been suggested that the

14 combinations of TIP3P-AMBER, TIP3P-CHARMM, TIP4P-OPLS and SPC-GROMOS

15 have been more relevant to the experiments[54,55]. Although there are some exceptions

16 shown in the literature, for instance see reference[55]. Becker et al[56] have listed a number

17 of considerations for choosing an appropriate force field in material science and

18 engineering and these suggestions also holds well for biomolecular simulations.

20 Solving Newton's equations of motion analytically is unpractical for the thousands of 21 degrees of freedom typically involved in many MD problems. As a result, numerical

22 integration algorithms, such as Verlet integrator[57], velocity Verlet integrator[58] and

23 leapfrog integrator[59], are usually employed to solve these equations and predict the next

24 move for all atoms during MD simulations. Since the dynamics of the covalent bonds

25 involving hydrogen atoms are not very crucial in biological problems, they are usually

26 constrained using integration algorithms, such as SHAKE[60], RATTLE[61] and

27 LINCS[62]. Hence, a time step value in the range of $1.5 \mathrm{fs}$ to $2 \mathrm{fs}$ is possible and has shown

28 to be suitable for MD simulations of many biological systems[46].

30 The main advantage of MD approach is its abilities to mimic the experimental conditions

31 in which a typical biological question is addressed. For instance, experiments are carried 
1 out by controlling different factors, such as temperature, pressure, number of atoms, ionic

2 concentration and the type of solvent used to solvate the interacting molecules. All these

3 factors can be readily adjusted and controlled in MD simulations within the context of

4 statistical mechanics ensembles [63]. These ensembles include the microcanonical

5 ensemble (constant total energy), canonical ensemble (constant temperature), and

6 isothermal-isobaric ensemble (constant temperature and pressure). The microcanonical

7 ensemble is the most basic approach and involves a constant number of particles $(\mathrm{N})$, a

8 constant volume (V) and constant energy (E). However, as the condition of maintaining a

9 constant total energy is not realistic [64], the canonical ensemble (NVT)[65] and

10 isothermal-isobaric ensemble (NPT) [66] are commonly used. A number of thermostats and

11 barostats, such as Langevin[67], Berendsen[68] and Nose-Hoover[69,70], have been

12 developed to fix the temperature and pressure in MD simulations. In fact, the isothermal-

13 isobaric (NPT) ensemble is the most widely used ensemble in MD simulations, as it reflects

14 the actual experimental conditions. There are a number of classical MD programs,

15 including but not limited to, AMBER (www.ambermd.org), CHARMM

16 (www.charmm.org), NAMD (www.ks.uiuc.edu/Research/namd/), GROMACS

17 (www.gromacs.org), Desmond (www.deshawresearch.com) and Hyperchem

18 (www.hyper.com). Some important quantities that are frequently used when analyzing MD

19 trajectories are provided in BOX 1.

\section{MD simulations and protein flexibility}

22 The dynamic nature of proteins is a well-established phenomenon [71]. Proteins are very

23 flexible biological molecules that can adopt multiple conformational states in solution [18].

24 Very few of these conformations are able to bind efficiently to the ligands and/or other

25 systems in the environment. For example, certain configurations of proteins may adapt an

26 open state that keeps the channels accessible for water molecules and ligands to

27 bind/unbind freely $[72,73]$. On the other hand, in some other conformations of the same

28 proteins, the highly malleable loops may be blocking the channel partially or completely

29 thereby restricting ligand access. In addition, binding of the ligand may also lead to

30 conformational changes in proteins, from local reorganization of side-chains to hinge

31 dynamics of domains [40-42]. As a result, proteins often shift between different 
1 conformational states separated by low- and high-energy barriers in the free-energy

2 landscapes during chemical reactions. Histone deacetylase 8 (HDAC 8) is one of the best

3 examples for dynamic mobility in proteins. These unusual dynamics of HDAC8 have been

4 captured by at least 21 different experimentally-determined structures (PDB IDs: 1T64,

5 1T67, 1T69, 1VKG, 2W22, 2V5W, 2V5X, 3EW8, 3EWF, 3EZF, 3EZT, 3F06, 3F07,

6 3F0R, 3SFF, 3SFH, 3MZ3, 3MZ4, 3MZ6, 3MZ7, and 3RQD)[46,71,74]. By comparison

7 of all the reported experimental structures, it was found that an $11 \AA$ deep active-site pocket

8 of the enzyme changes between a broadly open conformation to a partially open state and

9 a fully closed structure[71,74]. Some experimental structures of HDAC8 also display an

10 extra pocket that lies parallel to the main pocket (Fig. 3). All these structures are proposed

11 to exist in equilibrium and are involved in ligand binding/unbinding, product release or

12 water transfers[46,71]. Furthermore, some proteins could have additional druggable

13 binding sites, which are cryptic in nature and have the potency to modulate the

14 functionalities of the concerned receptors allosterically. Such cryptic or allosteric binding

15 sites are usually not easily detectable in the ligand-free structures, as in TEM1 $\beta$ -

16 lactamase[75] and p38 MAP kinase[76] for instance, and require significant

17 conformational changes in the receptors to become visible. Hence, these sites are usually

18 not detectable from a single representative structure and requires large conformational

19 sampling to reveal them. One of the well-known success stories of MD in such applications

20 is with regards to the discovery of a novel-ligand binding trench in HIV-integrase enzyme.

21 In 2004, Schames et al[77] performed MD simulations of HIV-integrase enzyme along

22 with the docked ligand and discovered a novel ligand binding region, the trench. The

23 existence of this cryptic site was later also confirmed by X-crystallography. Subsequently,

24 scientists from Merck along with their collaborators performed intense experimental

25 research[78] on this novel binding site, which eventually led to the development of novel

26 anti-HIV inhibitors such as raltegravir[19].

28 It is therefore logical to use an ensemble of protein conformations in SBDD, instead of a

29 single representation. Nevertheless, due to high-costs and technical complexities,

30 experimentally-determined structures for different conformations are only available for

31 few proteins. As discussed in earlier sections, MD simulations are now being used to 
1 collect ensembles of protein structures for SBDD in order to close this gap. Under this MD

2 scheme, the target structure (obtained from PDB or computational modelling) is initially

3 subjected to large-scale MD simulations followed by root mean squared deviation (RMSD)

4 conformational clustering to accumulate all possible conformations of a typical protein

5 structure. Subsequently, statistical analysis methods, such as Principal Component

6 Analysis (PCA), are then employed to transform the original space of correlated variables

7 into a reduced set of independent variables comprising of the most vital dynamics of the

8 system[40-42,79]. This will result in an ensemble of protein structures that can be used in

9 docking-based virtual screening. This MD scheme to account for receptor flexibility is

10 popularly known as the 'relaxed complex scheme' (RCS)[42]. RCS has been successfully

11 employed in a number of studies[40-43]. For instance, we have employed extensive MD

12 simulations to conduct an ensemble-based virtual screening against the MDM2

13 protein[41], a main regulator for $\mathrm{p} 53$. We performed over $50 \mathrm{~ns}$ MD simulations of the

14 structure of MDM2 using the AMBER99SB force field and NAMD program and sampled

1528 distinct conformations of MDM2 for further virtual screening of several ligand

16 databases[41]. The 28 structures included twenty-two structures that comprised $\sim 75 \%$ of

17 the apo-trajectory, five structures representing $\sim 80 \%$ of the bound-trajectory and a single

18 MDM2 conformation from the MDM2-p53 crystal structure [41]. The study revealed that

19 MDM2 is a highly flexible protein and adopted distinct conformational changes[41], which

20 were effectively captured using MD simulations, as shown in Fig. $4 \mathrm{a}^{2}$. In another study,

21 Bowman et al[80] performed MD simulations of p53-MDM2 complex and generated

22 multiple structures of the systems, so as to account protein flexibility in their subsequent

23 docking-based virtual screening. This led to the discovery of five small-molecule inhibitors

24 of the human MDM2-p53 interaction. Particularly, one of the compounds exhibited a Ki

25 of $110 \pm 30 \mathrm{nM}[80]$. These small molecules indeed have distinct scaffolds from nutlin, a

26 known inhibitor of MDM2-p53 interaction[80]. Thus, incorporating RSC approach is able

27 to discover novel therapeutically attractive small molecules. In one of our another studies,

28 we used the MD-based RSC approach to develop a computational atomistic model of a

\footnotetext{
${ }^{2}$ Reprinted from Journal of Molecular Graphics and Modelling, 28 (6), Khaled Barakat, Jonathan Mane, Douglas Friesen, Jack Tuszynski, Ensemble-based virtual screening reveals dual-inhibitors for the p53-MDM2/MDMX interactions, 555-568, Copyright (2010), with permission from Elsevier.
} 
1 human ether-á-go-go-related (hERG) ion channel [40]. Conformational sampling of the

2 MD trajectory of hERG resulted in 45 different clusters that made a comprehensive 3 description of backbone (Fig. 4b) and side-chain dynamics (Fig. 4c) of the inner cavity of

4 the ion channel[40] $]^{3}$. This model serves as a powerful tool to predict $\mathrm{hERG}$ blocking and

5 can be useful in developing safer and more efficient drugs[40].

7 In combination with other computational approaches, MD simulations can help in 8 characterizing protein-protein interactions. These types of interactions play important roles 9 in several biological processes, including signal transduction, cell metabolism and/or 10 transport. Understanding these interactions can access a new era of drug discovery, hence, 11 expanding the target space for new and more effective drugs[81]. Although the protein12 protein interfaces are generally large, only selected subset of residues are responsible for 13 the strong binding at these sites. Such residues, along with the surrounding domains, are

14 known as hot spots. Protein-protein interactions are also known to possess transient binding 15 pockets that are not captured in experimentally-determined structures. MD simulation has

16 become routine in approaches for identifying these hotspots and predicting binding sites

17 for their regulation. For instance, MD simulations have provided a detailed understanding 18 of the dimer interface in the HIV 1 protease enzyme, which is characterized by solvent 19 accessible surface areas and inter-dimeric hydrogen bonds[82]. In a recent study [83], we 20 employed MD simulations to model and characterize the human programmed death-1 (PD21 1) bound to its two human ligands, PDL-1 and PDL-2. Table 1 lists some of the studies 22 that have employed MD simulations for various applications (such as accounting protein 23 flexibility and dynamics, post-docking structural refinements and free energy of binding 24 calculations) on different target enzymes (or proteins) in the past five years.

\section{MD simulations and post-docking structural refinements}

27 Although docking can predict the optimal placement of a ligand within a receptor's active 28 site, not all of the key interactions between the ligand and receptor are usually depicted

\footnotetext{
${ }^{3}$ Reprinted from Toxicology Letters, 230 (3), Anwar Anwar-Mohamed,Khaled H. Barakat, Rakesh Bhat, Sergei Y.Noskov, D.Lorne Tyrrell,Jack A. Tuszynski,Michael Houghton, A human ether-á-go-go-related (hERG) ion channel atomistic modelgenerated by long supercomputer molecular dynamics simulations and its use in predicting drug cardiotoxicity, 382-392, Copyright (2014), with permission from Elsevier.
} 
1 accurately. Hence, it is generally recommended to perform MD simulations on the

2 complexes obtained from docking as this can help in optimizing their interactions. For

3 instance, in a previous study[84], molecular docking predicted that sulphonamide

4 derivatives bind effectively into the active site of aldose reductase, which was contrary to

5 the less activity found for these compounds in experiments. In silico refinements of these

6 structures using MD revealed that a water molecule from the exterior migrated to the

7 binding site and interrupted the key interactions between sulphonamide ligands and the

8 receptor. This was identified to be a reason for the reduced activity of the tested

9 compounds in experiments [84]. In another study, MD simulations were used to discern

10 the different docked complexes of propidium and human acetylcholinesterase based on

11 their stability[85]. The most stable structures identified with the help of MD simulations

12 were in excellent correlation with the binding modes proposed by experiments[85].

13 Similarly, a combination of ensemble-based molecular docking and MD refinements of

14 post-docking complexes helped us reveal for the first time a unique symmetrical binding

15 mode of Daclatasvir (a drug in phase III clinical trials) with the Hepatitis C virus (HCV)

16 NS5A protein and for different HCV genotypes[43], refer to Fig. $5^{4}$.

18 MD has made significant contributions in the understanding the structure-properties of G-

19 protein coupled receptors. For instance, a previous study involved post-docking MD

20 simulations to reveal significant dynamic changes in the human CC chemokine receptor 3

21 (CCR3) and the human muscarinic acetylcholine receptor 3 (hM3R) that influence their

22 ligand binding modes [24]. Especially, MD simulations found a strong H-bond between

23 the docked ligand and N508 residue of hM3R that is key to holding the complex. This was

24 again confirmed by performing MD simulations of N508A mutant hM3R and ligand

25 complex, in which the ligand was found to be pushed to the exit [24]. In another study by

26 Perdih et al[86], the authors employed molecular docking and MD simulations, along with

27 a range of experiments, and identified some of the furan-based benzene mono- and

28 dicarboxylic acid derivatives as potential inhibitors of all four bacterial Mur ligases. The

\footnotetext{
${ }^{4}$ Reprinted with permission from Journal of Chemical Information and Modeling, 55 (2), Khaled H. Barakat, Anwar Anwar-Mohamed, Jack A. Tuszynski, Morris J. Robins, D. Lorne Tyrrell, and Michael Houghton, A Refined Model of the HCV NS5A Protein Bound to Daclatasvir Explains Drug- Resistant Mutations and Activity against Divergent Genotypes, 362-373. Copyright (2015) American Chemical Society.
} 
1 authors initially performed in-vitro testing of seven furan-based benzene-1,3-dicarboxylate

2 derivatives, based on their previous studies and found out that one of the compounds was

3 able to inhibit all Mur ligases in the mircromolar range[86]. Subsequently, this compound

4 was docked into the active site of MurD enzyme and two different ligand binding modes

5 were identified. Subsequently, the authors performed $\sim 20 \mathrm{~ns}$ long MD simulations and

6 interaction energy calculations, so as to further refine the post-docking complex and also

7 identify the best binding mode of the ligand. Finally, based on the results obtained, four

8 novel furan-based benzene-monocarboxylic acid class were discovered to inhibit multiple

9 Mur ligases in the low micromolar range[86]. Moreover, one of the newly discovery

10 compounds also exhibited promising antibacterial activity against $S$. aureus[86].

12 Previous studies[87-89] have also shown that integrating induced-fit docking (IFD)

13 method along with $\mathrm{MD}$ and/or $\mathrm{QM} / \mathrm{MM}$ simulations can be useful for the efficient

14 description of induced-molecular flexibilities within the protein-ligand complexes and also

15 for accurate binding mode analysis of ligands. For example, in a recent study, Distinto et

16 al[87] employed IFD and MD simulations in order to unravel the putative binding modes

17 and activities of 1-(aryliden-2-(4-(4-chlorophenyl)thiazol-2-yl)hydrazines against

18 monoamine oxidase B (MAO-B) enzyme, an attractive target for neurodegenerative

19 diseases. By structural alignment of several X-ray structures of MAO-B co-crystalized with

20 different inhibitors, it was understood that the enzyme adopted induced-fit changes with

21 respect to the bound ligands. Hence, the authors initially performed IFD using the

22 Schrodinger drug discovery suite, during which the side chains near the inhibitor were kept

23 flexible. The results from the IFD showed explained the how ligand binding tend to induce

24 structural changed in the protein. However, many of the compounds showing two binding

25 modes were ranked high in IFD. In order to find out the best binding mode of the inhibitors,

26 the authors performed 3 - 5 ns long MD simulations for both the binding modes of two of

27 the top-ranking compounds from IFD. The MD results followed by the free energy

28 calculations highlighted the significance of fluorine atom interacting with the water near

29 the cofactor and the influence of steric bulkiness of substituents in the arylidene moiety.

30 The authors propose that the pharmacophore features of these experimentally synthesized

31 compounds, developed using combined IFD, MD and free energy calculations, should be 
1 useful for achieving novel high-affinity MAO-B inhibitors for the treatment of 2 neurodegenerative disorders[87].

4 In another study, Fu et al[88] combined IFD with classical MD simulations, free energy of

5 binding calculations and $\mathrm{QM} / \mathrm{MM}$ calculations to study the substrate binding to human

6 biliverdin-IX $\alpha$ reductase (hBVR-A) of biliverdin-IX $\alpha$ and four analogues. hBVR-A is a

7 key enzyme in regulating a wide range of cellular processes and is involved in the

8 conversion of biliverdin-IX $\alpha$ to bilirubin-IX $\alpha$. In this work[88], the authors initially

9 employed the structure of the hBVR-A/NADPH/substrate I complex for the docking of

10 analogs into the binding pocket using the IFD procedure implemented in the Schrodinger

11 program. During the IFD, a tyrosine residue in the active site was treated with flexibility.

12 Subsequently, the best-ranking ternary complex structures from IFD were subjected to

13 classical MD simulations[88]. Multiple snapshots obtained from the MD simulations were

14 used for performing free energy of binding calculations. The predicted free energies of

15 binding for five analogues agreed well with the experimental binding affinities and also

16 helped to identify the best binding pose for the complexes[88]. Finally, the authors

17 investigated the catalytic mechanisms of the ternary complex structure (in this study) by

18 calculating the reaction energy profiles using advanced QM/MM calculations. These

19 advanced calculations were useful to understand the reaction mechanisms of the system

20 studied, which on the long-run should assist in the design of potent hBVR-A inhibitors[88].

21 Thus, MD serves as an important tool for not only refining the post-docking complexes,

22 but also for revealing more appropriate binding modes of the ligands within the receptor 23 structures.

25 MD simulations and predicting the free energy of binding

26 Molecular recognition is critical in several biochemical and biological processes [90].

27 Many biological processes are initiated by specific binding between two interacting entities

28 in the cell. Although docking, combined by MD simulations, can provide a clear image of

29 the shape complementarity between these entities at their binding interface, whether these

30 interactions are significant or realistic requires an additional and essential piece of

31 information, namely the free energy of binding, which is the driving force toward forming 
1 this complex. Calculation of the binding free energy $\left(\Delta G_{b i n d}\right)$, i.e., the free energy

2 difference between the ligand-bound state (complex) and the corresponding unbound states

3 of proteins and ligands, is used to quantify the affinity of a ligand to its target. Assessing

4 the $\Delta \mathrm{G}_{\text {bind }}$ of a series of ligands against a particular target can discern those ligands with

5 higher binding affinities with the target. The $\Delta \mathrm{G}_{\text {bind }}$ calculations are thus very important in

6 drug design, and normally follow the docking-based virtual screening processes. A number

7 of computational methods, from computationally rigorous thermodynamics pathways

8 approaches to less complex end-point methods, have been developed for $\Delta \mathrm{G}_{\text {bind }}$

9 calculations. The former methods include thermodynamic integration (TI) and free energy

10 perturbation (FEP) methods; while liner interaction energy (LIE), Molecular Mechanics-

11 generalized Born surface area (MM-GBSA), and Molecular Mechanics-Poisson-

12 Boltzmann surface area (MM-PBSA) are end-point methods. Each of these methods has

13 its own strengths and limitations, and their computational requirements and speed are

14 inversely correlated with their accuracy.

16 TI and FEP methods are thermodynamic pathways approaches that are commonly 17 employed for the calculation of relative binding free energies[91,92]. These methods are 18 mainly based on the application of thermodynamic cycles and thus require the 19 transformation of the system from the initial state to the final state through alchemical 20 changes of the system energy function[91]. These methods involve change of a ligand A 21 into ligand B in two states, such as solvent-only unbound state (of the ligands) and bound 22 state (i.e., ligand-protein complexes). This will provide free energy changes for the 23 unbound states ( $\Delta \mathrm{G}_{\text {unbound }}$ ) and bound states ( $\Delta \mathrm{G}_{\text {bound }}$ ) of the ligands [91]. It is also possible 24 to mutate ligand A to "nothing", which in principle can provide absolute free energies of 25 binding. Understandably, these methods (TI and FEP) demand multiple MD simulations 26 and rigorous sampling of ligand, protein and solvent degrees of freedom. As a result, the 27 thermodynamic pathways methods are in general able to provide very accurate estimation 28 of the free energy of binding at the cost of high computational time[93,94]. For instance, 29 the TI method coupled with MD simulations has been employed to identify potential 30 huperzine derivatives with higher binding affinity towards the acetylcholinesterases[95].

31 Similarly, the FEP approach has also shown to predict more accurate binding free energies 
1 for a number of inhibitor-enzyme complexes[93,96]. However, estimating the $\Delta \mathrm{G}_{\text {bind }}$

2 values using these methods require large numbers of conformational samples, which in

3 turn inflate the computational costs heavily[93,94]. Given the need for enormous

4 computational resources, these methods have mostly been applied for only small sets of

5 ligand-protein complexes. Nevertheless, with increasing supercomputing capabilities and

6 more improved methods, TI and FEP are gradually being involved in the SBDD pipeline,

7 especially in guiding lead optimization[92,97-100]. For instance, a recent work[100] by a

8 large team of authors, from Schrödinger, Nimbus, Columbia, Yale, and UC-Irvine, show

9 that FEP calculations are able to make highly accurate affinity predictions across a wide

10 range of ligands and targets. This work included fairly diverse sets of targets, such as

11 BACE, CDK2, JNK1, MCL1, p38, PTP1b, Tyk2, and thrombin. The estimated binding

12 free energies reported in this study were in very good agreement with the experiments.

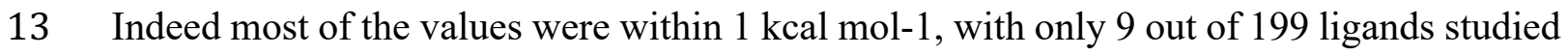

14 deviated from their experimental values by over $2 \mathrm{kcal} \mathrm{mol}^{-1}[100]$.

16 A less rigorous alternative to thermodynamic pathways is the end-point approaches, which

17 include methods such as LIE, MM-PBSA, and MM-GBSA. Unlike thermodynamic

18 pathways approaches, these end-point methods sample only structures involved at either

19 ends of the reaction pathways; that is, the free receptors (proteins) and ligands and the final

20 ligand-protein complexes. The $\Delta \mathrm{G}_{\mathrm{bind}}$ in this approach can be written as,

$$
\Delta G_{\text {bind }}=G_{\text {complex }}-\left(G_{\text {protein }}+G_{\text {ligand }}\right)
$$

23 The LIE method, developed by Aqvist et al[101], considers the process of a ligand $(L)$

24 binding to a protein as a partition problem, in which the free ligand $(F)$ from the bulk 25 solvent medium is transferred to a solvated protein environment $(P)$. Therefore, two 26 independent MD simulations, one for the complex and the other for solvated ligand, needs 27 to be performed in order to calculate $\Delta \mathrm{G}_{\text {bind }}$ using the LIE method. Nonetheless, the reliance 28 of the LIE on the end-points of binding makes it an attractive (and affordable) approach 29 for lead optimization in drug discovery. A number of studies have employed LIE method 30 for the computational analyses of inhibitors against a variety of targets. This includes 
1 benzamide-based thrombin inhibitors[102], inhibitors of Mycobacterium tuberculosis

2 H37Rv cytidine deaminase[103], sertindole analogues to block hERG potassium

3 channel[104] and allophenylnorstatine molecules to inhibit Plm4 enzyme, a target for

4 Plasmodium malariae[105], for instance. The LIE method has been shown to predict

5 binding free energies with a root mean square error (RMSE) $<1 \mathrm{kcal} \mathrm{mol}^{-1}$ compared to

6 the experimental values[106]. As indicated above, the thermodynamic pathways methods

7 are also able to make predictions with a similar, if not better, accuracy range for different

8 targets.

10 MM-GBSA and MM-PBSA are the two other well-established end-point methods that are

11 most popular in SBDD. The two methods employ an implicit solvent model to account for

12 the solvent molecules and use dielectric continuum models to obtain the electrostatic

13 components for the solvation energy. The MM-PB(GB)SA $\Delta \mathrm{G}_{\text {bind }}$ can be estimated as,

$$
\Delta G_{\text {bind }}=\Delta E_{M M}+\Delta G_{S o l v}-T \Delta S
$$

16 Here, $\Delta E_{M M}$ refers to the molecular mechanical energy and it is the sum of all energies

17 from the bonded and non-bonded interactions. The solvation energy, $\Delta G_{s o l v}$, is the sum of

18 the polar and non-polar contributions of solvation. The polar solvation terms $\left(\triangle G_{P B / G B}\right)$ are

19 estimated using a Generalized-Born model or a Poisson-Boltzmann solver. The non-polar

20 contributions are computed based on the size of the solvent-accessible surface area

$21\left(\Delta G_{S A S A}\right)$ in the ligand and protein. The final component of the $\Delta G_{b i n d}$ equation (equation

22 7) is $\mathrm{T} \Delta S$, which corresponds to the conformational entropy changes in the reaction-

23 product (i.e., protein-ligand complex), upon ligand binding.

25 The inclusion of conformational entropy $(\mathrm{T} \Delta S)$ in the $\mathrm{MMPB}(\mathrm{GB}) \mathrm{SA}$ calculations, in order 26 to obtain absolute $\Delta G_{b i n d}$, remains challenging. The accurate calculation of (T $\left.\Delta S\right)$ is

27 computationally expensive and in many cases, its inclusion does not guarantee better 28 accuracies in the final energies[46,107,108]. Rather, previous studies have shown that 29 accounting for conformational entropy obtained from insufficient MD sampling has 30 adversely affected the calculations[107,109]. For instance, Su et al[107] showed that the 
1 accuracies of their MM-PBSA and MM-GBSA calculations for 16 known benimidazole

2 inhibitors against F.tularensis Enoyl-ACP Reductase were significantly affected because

3 of using different number of frames for their enthalpy and entropy calculations. The authors

4 sampled 2400 frames from the MD trajectory used for their enthalpy calculations; however,

5 due to limited computational resources, they only used 48 frames (evenly selected from the

6 trajectory) for the entropy calculations. Therefore, it is important to have large numbers of

7 MD snapshots to derive a reliable estimate of absolute $\Delta G_{\text {bind, }}$, which can significantly

8 increase the computational costs. As a result, many studies tend to neglect T $\Delta S$ and use the

9 'relative' $\Delta G_{\text {bind }}$ instead. Relative $\Delta G_{\text {bind }}$ energies can be predicted with a reasonable

10 accuracy, and are generally sufficient to rank a group of compounds against the same target

11 in SBDD [110].

13 Two strategies are commonly employed in MM-GBSA and MM-PBSA calculations: (i)

14 the three-trajectory scheme and (ii) the single trajectory scheme[20,46,107,111]. In the

15 former, three different MD trajectories that pertain to the 'apo' protein, free ligand and the

16 ligand-protein complex (i.e., the end-point structures) are sampled for snapshots. In

17 principle, this three-trajectory scheme provides more accurate results than the single-

18 trajectory approach; however, it demands high computational costs [110,111]. On the other

19 hand, the single trajectory scheme requires only a single MD simulation for the ligand-

20 protein complex, which significantly reduces the required computational time [20,110-

21 112]. Apart from the choice of strategy, there are a number of factors that may affect

$22 \operatorname{MMPB}(\mathrm{GB}) \mathrm{SA}$ calculations, which includes length of simulations, choice of the force

23 field, solute dielectric constants, solvent model, and the net charge of the systems. For

24 instance, it has been argued that employing multiple short and independent MD

25 simulations, instead of one long MD trajectory, can provide better $\Delta G_{b i n d}$ predictions

$26[107,108,111,113]$.

28 There have been a number of studies that compared and tested the efficiencies of MM-

29 GBSA and MM-PBSA in predicting accurate $\Delta G_{b i n d}$ energies for different ligand-protein

30 complexes. Their general conclusion is that the accuracy of these methods tends to be

31 system-dependent. For example, Hou et al.[110] found MM-GBSA to predict accurate 
1 relative $\Delta G_{\text {bind }}$ for 59 ligands against six different protein targets in their study, when 2 compared to MM-PBSA that outperformed the former in making the absolute $\Delta G_{\text {bind }}$ 3 predictions. We have extensively applied MM-PBSA method for a range of studies, 4 including screening and ranking of ligands against ERCC-XPA complex[41], 5 understanding the binding mode of daclatasvir onto the NS5A viral protein [43] and

6 binding of human programmed death-1 of t-cells with its ligands[83]. On the contrary, 7 Oeheme et al[109] concluded that MM-GBSA performed better than the MM-PBSA in 8 computing $\Delta G_{\text {bind }}$ of their ligand-HIV protease systems. Thus, it is clear that neither of 9 these two methods is universally superior and the choice of the method should be made on 10 a case-by-case basis. For example, Jordheim et al[114] combined, MD simulations, virtual 11 screening and MM-PBSA based binding-free energy calculations, along with different 12 experimental techniques, to identify potential inhibitors of ERCC1-XPF protein-protein 13 interactions. The authors performed 20 independent virtual screening runs against the 20

14 XPF structures present in an NMR ensemble, after their MD equilibration. Top hits from 15 each screening were extracted and then ranked them based on their binding free energies.

16 From these results, 73 compounds were subjected to a range of experiments, including 17 cytotoxicity assays, steady-state fluorescence and synchronous fluorescence experiments, 18 and immunocytochemistry. The hits were evaluated on A549 and HCT116 cancer cells. 19 Finally, one compound was found to exhibit promising activity in all the experiments and 20 was also able to interact with the domain of XPF that is responsible for interacting with 21 ERCC1, thus disrupting the protein-protein interactions. Thus, MD-based binding free 22 energy calculations are helpful in guiding the hit identification stage. However, one of the 23 significant drawbacks of both these methods is their inability to make accurate predictions 24 for ligands with formal charges[109,111,115]. Hence, it is important to improve the 25 existing methods or develop new methods which can account for charged ligands 26 (including tautomers), which form a significant area of drug research.

\section{MD simulation and solvent dynamics analyses}

29 Computational analyses of structure and thermodynamic properties of water have recently 30 become a useful tool in SBDD[116-119]. The properties of surface water molecules have 31 been proposed to play important roles in molecular recognition and ligand-protein (and/or 
1 protein-protein) interactions in solution[116,120]. Though small in size, water molecules

2 are involved in a range of interactions, including H-bonds and van der Waals contacts[120].

3 Due to such interactions, it is often difficult to displace water molecules to facilitate the

4 binding a drug. The energy involved in relocating water molecules between surface layer

5 and bulk water, upon binding of a macromolecule (protein for instance) with another

6 macromolecule and/or ligands, therefore, can significantly impact the overall free energy

7 of binding[116-120]. Hence, the hydration patterns of a binding pocket can offer important

8 insights into the properties of the pocket and also quantify the hydrophobic forces involved

9 in the binding of small-molecule drugs with proteins. There are a number of in silico tools

10 that can help in extensive molecular descriptor analyses of solvation from the MD

11 trajectories. These algorithms include WaterMap (from Schrodinger)[121], WaterFLAP

12 (from Molecular Discovery)[122], SZMAP (from OpenEye)[123], GIST (in

13 Amber)[120,124], WatMD (in-house tool of Novartis Inc)[116,125], SPAM (from

14 GlaxoSmithKline)[126], STOW[127], WatClust[127], etc. Some of these methods,

15 WaterMap[121], STOW[127] and WatClust for instance, are based on inhomogeneous

16 fluid solvation theory (IST), where enthalpy is accounted directly from non-bonded

17 interactions and entropy is calculated from a local expansion in terms of correlation

18 functions[121].

20 For instance, the WaterMap program[121] initially clusters the water molecules (in the MD

21 trajectory) based on their spatial distribution such that they form individual hydration sites.

22 Subsequently the hydration sites are analyzed using IST to determine the enthalpy and

23 entropy properties of water molecules within each site. This method has been successfully

24 employed to gain insights into binding sites for various systems, including peptides binding

25 to PDZ domains[128], the FKBP12 protein[129], protease and kinase binding 26 affinity[130,131] and the A2A GPCR[132]. For example, Beuming et al[129] employed

27 WaterMap tool to analyze the hydration sites for a panel of twenty-seven different protein

28 targets across a range of families. Initially, the authors[129] performed $\sim 2 \mathrm{~ns}$ long MD

29 simulation for each of the targets and the resultant trajectories were subjected to analyses

30 with the WaterMap program. The results[129] revealed $\sim 31,500$ hydration sites in the

31 targets, for which the authors calculated their thermodynamic information (such as free 
1 energy, entropy and enthalpy). The authors further demonstrated that such thermodynamic

2 properties of the hydration sites could be used to identify potential binding sites and

3 evaluate their druggability[129]. It was found that clusters of high-energy solvation sites

4 mostly inclined to be related with binding sites. However, Ramsey et al[120] notes that the

5 IST-based methods are limited to the analyses of high-occupancy hydration sites and they

6 do not describe significantly the hydration structures in low-density regions. As an

7 alternative to these methods, the authors developed a tool named grid IST (or GIST)[120]

8 and implemented it into CPPTRAJ toolset of AmberTools. GIST discretizes the integrals

9 of IST onto a 3D-grid, which fills the binding pocket region and thus covering both high-

10 density and low-density regions[120]. As a result, unlike the IST methods, the GIST is able

11 to offer a smoothed map of water structure and the corresponding thermodynamic

12 information for the complete region of interest. For instance, GIST analyses of the

13 molecular host cucurbit[7]uril have revealed significant information about the hydration

14 structure and thermodynamic properties in this receptor[124]. The results particularly

15 revealed a toroidal region of high density hydration site at the center of the host's nonpolar

16 cavity. The results[124] also show that this specific hydration site, despite having high

17 density of water molecules, is energetically and entropically not favorable. The authors

18 relate this to the known ability of this receptor to bind external molecules with unusually

19 high affinities[124]. Henceforth, a combination of MD simulations and explicit analyses

20 of solvent dynamics are helpful to advance our knowledge about the effects of water

21 molecules in structural biology and drug design[124].

22

\section{Constant pH molecular dynamics}

24 The ligand-protein complex formation not only leads to conformational changes in the

25 structures of the proteins and/or ligands, but can also impact the $\mathrm{pK}_{\mathrm{a}}$ values of their charged

26 side chains. The most common practice in molecular docking and standard molecular

27 dynamics is to assign fixed protonation states for the protein residues, substrates and

28 ligands, based on prior chemical knowledge. However, it is a known fact that the

29 protonation states of a typical ionisable group involve dynamic processes that can alter the

30 chemical environment during binding. Previous studies[133,134] noted that the $\mathrm{pKa}$ values

31 of titratable residues may change due to a number of factors. This includes the solvation of 
1 the group upon ligand-binding, electrostatic interactions between the ligand and protein,

2 and structural reorganizations within ligand-protein complexes after binding. Thus the

3 protonation states of ionisable amino acid residues and non-protein molecules (ligands and

4 substrates) may be subjected to a change during the course of MD simulations. By

5 preserving the protonation states, the $\mathrm{MD}$ simulations ignore any binding-induced $\mathrm{pK}_{\mathrm{a}}$

6 changes within the systems. This missing information can limit our complete

7 understanding of the underlying biological processes.

9 Constant $\mathrm{pH}$ molecular dynamics (or $\mathrm{CpHMD}$ ), has been developed for the computational

10 prediction of $\mathrm{pKa}$ values[135] for ionisable residues in the biological systems under study

11 (refer to Box 2). The early CpHMD approach employed GB solvent as the continuum

12 aqueous environment and Langevin dynamics for the propagation through the non-solvent

13 (or solute) trajectories [136]. However, this approach has been found not so accurate for

14 many systems, particularly, when water molecules play an active role. Alternately, Donnini

15 et al[137] developed a fully atomistic CpHMD method with $\lambda$-dynamics approach, which

16 can be carried out in explicit solvents. This method allows for the dynamic change of

17 protonation states of titratable groups, thus being able to predict the possible average

18 protonation states at a given $\mathrm{pH}$. This method samples the relevant configurations of the

19 end states of titration groups, by considering the protonated as $\lambda=0$ and deprotonated as

$20 \lambda=1[137]$. Given the importance of the protonation states of titratable groups in SBDD, it

21 is suggested that a constant $\mathrm{pH}$ MD simulation be performed for the ligand-protein

22 complexes before any production MD simulations are initiated. This way, the protonation

23 states of the ionisable groups in the system can be accurately described.

25 More recently, there have been significant developments in improving the CpHMD [138-

26 141]. For instance, attempts have been made to improve CpHMD using the replica

27 exchange concept (vide supra). The basic idea is to perform simulations of different

28 replicas at different $\mathrm{pH}$ values. After some set number of steps, the $\mathrm{pHs}$ are exchanged

29 between the replicas so as to sample a wider range of protonation states[139]. This

30 approach has been shown to greatly improve the convergence rate and accuracy of $\mathrm{CpHMD}$

31 simulations[140]. 


\section{Limitations of MD}

3 Classical MD simulations remain a valuable tool in drug design. They are helpful in

4 understanding key molecular motions, energetics, ligand-protein interactions, receptor

5 flexibilities, and conformational changes in the molecular systems, which facilitate the

6 identification of potential candidates with higher affinities to targets. However, it is also

7 important to acknowledge that MD also has some potential limitations and pitfalls, most

8 particularly those concerning time limitations, force-field issues and quantum-

9 effects[53,142].

\section{Time limits and the sampling problem}

12 At present times, typical MD simulations are carried out on systems containing hundreds

13 to millions of atoms, and for several nanoseconds to microseconds timescales. Although

14 these are impressive developments in the field (of MD), it is possible that such time limits

15 may not be sufficient to relax the systems to study certain quantities. For instance, a number

16 of physical properties of biological systems, such as protein folding, ligand binding and

17 unbinding processes mostly occur at large timescales that are normally inaccessible using

18 classical mechanics MD simulations. Furthermore, it is known that biological systems can

19 get trapped in deep energy wells of their potential energy surfaces[143], which may result

20 in sampling of insufficient and/or non-relevant conformations even from long MD

21 trajectories[144]. Improper preparation of the initial structure or insufficient equilibration

22 of the initial structure(s) can impact the quality of the MD results. Sampling (or)

23 equilibration of an ensemble of structures, therefore, remains one of the key issues in MD

24 simulations. Such challenges can be tackled by employing alternative strategies. One of

25 the solutions is to apply an enhanced sampling MD approach[46,145], in which an

26 additional bias, such as an external force, is applied to the system in order to explore the

27 different potential energy surfaces. Although this strategy introduces some artefacts from

28 external bias, it is useful to allow large-scale conformational changes in the systems within

29 the affordable computational cost. Several enhanced sampling approaches have been

30 developed, including metadynamics, replica exchange molecular dynamics (REMD),

31 random acceleration molecular dynamics (RAMD), steered molecular dynamics (SMD) 
1 and adaptive bias force steering (ABFS). There are a number of reviews, for example see

2 references[145-147], discussing the applications of these methods in SBDD. Alternatively,

3 coarse-grained MD (CG-MD)[148], which reduces the degrees of freedom in large

4 systems by clustering groups of atoms into CG beads, has been developed to deal with

5 large dynamic changes in more complex macromolecules.

7 Force field issues and quantum effects

8 Molecular mechanics force field employed in the simulation plays vital roles in defining

9 the structural model of the studied system. Force fields are usually developed by combining

10 available experimental data and the results from high-level ab initio calculations on small

11 models that form larger systems, and hence they are fundamentally

12 approximations [53,142]. Furthermore, force fields are parameterized such that they include

13 several atom types describing varied situations of the same atoms (or functional groups).

14 Due to such reasons, the transferability of force fields is restricted. Thus, results of MD

15 simulations are reliable only as long as the potential energy functions (or force fields)

16 mimics the forces experienced by the atoms in the real system under study[142].

18 Classical MD, because of its capabilities to handle large-size systems using affordable

19 computational resources, has gained extraordinary popularity in SBDD. Classical

20 approximations are mostly well-suited for non-reactive molecular interactions in biological

21 systems[149,150]. However, they are not able to effectively describe the chemical

22 reactions occurring in biological systems. For example, classical MD may not be able to

23 offer great solution for understanding the reaction mechanisms of drug/substrate-protein

24 complexes, chemical processes of proton transfer within active site, and binding/cleaving

25 processes of certain covalently bonded ligands. In such cases, the use of quantum-

26 mechanics (QM), which explicitly models the electrons in the system, becomes essential

27 at the expense of computational time. In order to overcome this challenge, reactive force

28 fields have been developed recently that allows chemical reactivity to be treated to some 29 extent $[53,149,150]$. In reactive force fields, the interatomic potential defines chemical

30 reactions by implementing a bond-order formulation. Within this scheme, the bond orders

31 in the system are empirically calculated using interatomic distances between atoms during 
1 MD simulation. Whereas, the electronic interactions driving chemical bonding are treated

2 implicitly such that facilitating the modeling of changes in atom connectivity[149,150].

3 Recent review articles, for example see references [149,150], discuss various applications

4 and challenges of such reactive force fields.

6 Another important challenge faced by classical MD is accounting electronic polarization, 7 a significant quantum effect[142]. Within the classical MD framework, each atom in the 8 system is assigned with a pre-set partial charge and is maintained throughout the 9 simulation. Nevertheless, this is not always true, as the biomolecules are in general 10 polarizable; meaning that the electron clouds encircling the atoms constantly shift in 11 response to their chemical environment. Thus, it would be effective if the partial changes 12 could be represented as a dynamic parameter, which is not the case with most of the current 13 classical force fields. Realizing the importance of this challenge, there have been 14 significant ongoing efforts to develop robust polarizable force fields for MD 15 simulations[151]. Some of the current generation polarizable force fields include 16 AMOEBA[152,153], CHARMM Drude and AMBER ff02[151]. Indeed, it is important to

17 note that polarizable force fields also have their own challenges and should be used with 18 caution. For example, these polarizable force fields are in general slower than non19 polarizable force fields and, as a result, they are more vulnerable to sampling issues.

20 Therefore, polarizable force fields may not be suitable systems where large conformational

21 sampling plays important roles. Though having some weaknesses, the current polarizable

22 force fields are promising. Given the importance of electrostatic interactions in biological

23 systems, and with more developmental efforts underway, polarizable force fields will soon

24 become an inevitable choice for classical MD simulations in future. There are some recent

25 articles that discusses the current status and future directions for polarizable forces and MD 26 simulations [151-153].

\section{Advanced hybrid QM/MM MD}

29 Although there have been significant efforts to fix the issues (concerning chemical

30 reactivity and electronic polarization) within the classical MD framework, employing 31 quantum-mechanical (QM) MD, which explicitly models the electrons in the system, has 
1 been an alternative practical strategy in biomolecular simulations and SBDD. QM-MD

2 generates dynamical trajectories by using the forces obtained from the electronic structure

3 calculations that are performed at every time step of simulation. It is, therefore, able to

4 accurately describe any reactions involving significant electronic effects such as electron

5 correlation and electron polarization effects[154] [155]. Nevertheless, QM-MD

6 simulations are extremely computationally intensive, which limits the practicality of

7 applying this approach only to smaller sized systems ( $\sim 10^{2}$ atoms) and for limited time

8 scales $\left(\sim 10^{-12} \mathrm{~s}\right)[156]$. Hence, it was extremely important to find a mid-point that offers

9 both 'the chemical accuracy' of QM-MD and 'feasibility' of MM-MD. To address this

10 problem, Warshel and Levitt[157] introduced a state-of-the-art hybrid MD scheme

11 popularly known as $\mathrm{QM} / \mathrm{MM}$. In this approach, a chemically reactive region in ligand-

12 protein complex (mostly binding site residues and bound ligand) are treated with more

13 accurate QM methods, and the rest of the system is described using MM force fields (Fig.

14 6). To date, a number of QM/MM implementations have been developed[158-160] and

15 applied in many studies that focussed on large drug-protein and/or protein-protein systems.

16 For example, in their recent study, Chen et al[161] employed QM/MM MD and QM/MM

17 GBSA method for studying the interactions of benzamide inhibitors with trypsin. In this

18 study, the authors treated the active site residues of the receptor and the inhibitors with QM

19 methods (B3LYP/6-31G(d), PM3, PM6, and RM1) and the rest of the system with classical

20 ff99SB force field and AMBER program. The study found that binding free energies

21 calculated with the snapshots obtained from QM/MM MD trajectories displayed excellent

22 agreements with experimental values[161]. In another study[162], QM/MM MD

23 simulations revealed that the fourth ligand coordinating with the active site zinc ion in

24 Acutolysin A enzyme is a water molecule, rather than a hydroxide anion, correcting a

25 misconception from the low-resolution X-ray crystal structure. It was also revealed by a

26 study that the QM/MM FEP approach outperformed the conventional FEP scheme in

27 predicting accurate binding free energies for a set of fructose 1,6-bisphosphatase inhibitor

28 [93]. Cui and co-workers[163] showed that a hybrid QM/MM-FEP approach could be used

29 to predict accurate pKa values of biological systems. Thus, QM/MM MD simulations have

30 the ability to offer accurate dynamic information that is significant in understanding the 
1 structure-function relationships of proteins and their interactions with different classes of

2 ligands, the key in drug discovery research.

4 Nevertheless, it is also important to acknowledge the fact that QM/MM MD simulations

5 also have some clear pitfalls. One of the most important problems in QM/MM simulations

6 is the treatment of the interface region that connects the QM part with that for MM,

7 particularly if they are covalently bonded as in the case of ligand-protein systems. When a

8 complete system is explicitly cut into QM and MM parts, then it will leave the former

9 region with incomplete valences, which can lead to a failed QM treatment[164]. The most

10 common strategy to overcome this issue is to cap the bordering QM residues, which

11 underwent partition, with hydrogen atoms. But such hydrogen capping introduces new

12 atoms into the QM region those were not originally present in the real system, which may

13 lead to some artefacts[164]. Furthermore, QM/MM MD simulations of large protein-ligand

14 systems are still very computationally expensive. Hence, they can only be applied to select

15 systems in drug design, such as for those top-ranking hits filtered from thorough virtual

16 screening and classical MD simulations, where follow-up details about key ligand-protein

17 interactions for pharmacophore modelling are computationally justified.

\section{Perspectives on integrating the computational approaches}

20 Last decade has observed tremendous developments in the field of molecular modelling 21 and drug design methods. As discussed above, a number of modelling and MD-based 22 approaches are available to help in the modern drug design and discovery efforts.

23 Nevertheless, how we integrate these methods, along with other in silico approaches and 24 experiments, is important for increasing our chances of identifying more promising hits

25 from the chemical pool of compounds. Although there are no specific set of rules on how

26 these methods should be combined, extensive knowledge and experience gained over years

27 have provided some logical strategy of implementing them. In Fig. 7, we present a more

28 simplified and practical work-flow that assembles classical MD, binding free energy

29 calculations and $\mathrm{QM} / \mathrm{MM}$ methods at various stages leading from hit-identification to lead

30 optimization. For instance, the need of classical MD simulations could first arise upon

31 having one (or) more initial three-dimensional structures either from the PDB or through 
1 molecular modelling methods. Because most of these methods are single snapshots of the

2 target, long classical MD simulations (usually few hundred nanoseconds time scale) are

3 required so that large conformational changes in the target can be captured during the

4 simulation. At this stage, the user needs to make a number of cautious choices, such as the

5 software program, the empirical force-field and simulation parameters that are suitable to

6 perform stable MD simulations.

8 Following this stage, different clustering algorithms, such as RMSD-based clustering or

9 PCA analyses, can be performed to sample the dominant conformations of the target from

10 the MD trajectory. The target conformations obtained from the MD simulations will serve

11 the purpose of addressing the protein flexibility concerns during the subsequent virtual

12 screening procedures. Indeed, there are some computational methods to identify possible

13 cryptic binding sites from these ensemble of target structures and target them during

14 screening. Following the docking and scoring, MD simulations can again be performed on

15 the ligand-protein complexes, in order to refine the complexes and calculate their binding

16 affinities. At this point, the user must make a number of careful selections, including the

17 length of MD simulations, force-field for simulation and methods for binding-free

18 estimations. Usually, it is suggested that short MD trajectories ( 1-2 ns long) are collected

19 for each ligand-target complexes and use them for free energy of binding calculations. The

20 end-point methods, MM-PBSA or MM-GBSA, are mostly popular for these calculations;

21 although other methods, such as FEP, are gaining popularity in the field. Once the high-

22 ranking compounds are identified, they can be experimentally tested using different kinds

23 of assays. At the current stage, it is hoped that a $5-10 \%$ of hit rate (during experimental

24 testing) can be achieved by incorporating rigorous computational modelling and pre-

25 screening protocols; although this might not be the case always.

27 Whatever the results from the experiments are, positive or negative, they can be back-fed

28 to the computational protocol so as to improve it for subsequent phases of screening. For

29 example, if the results are negative (meaning no significant hits were identified), then

30 probably the lengths of initial MD simulations can be increased so as to increase the sample

31 size for target conformations; and/or increase the chemical search space by increasing the 
1 numbers of compounds in the libraries; and/or refine the parameters in the docking 2 protocols; and/or increase the length of MD simulations for binding free energy 3 calculations; and/or even change the method used for free energy estimations. In the event 4 of obtaining good hits from the experiments, then the user might wish to perform extended 5 MD simulations (now for hundreds of ns) to understand the key dynamic interactions 6 between the hits and the targets. Binding free energy methods (or) other enhanced sampling 7 MD methods can also be applied at this stage so as to gain in-depth knowledge about 8 binding mode(s) of the hits. Based on these information, an effective pharmacophore model 9 or QSAR model (and/or experimental SAR) can be developed and implemented in

10 subsequent screening protocols. When one or more promising hits, those showing attractive

11 inhibition potentials, promising immunological activity and also non-toxic profiles, are 12 identified, then complexes of such hits can be taken forward for more advanced and 13 computationally expensive $\mathrm{QM} / \mathrm{MM}$ simulations. At this stage, the user must be cautions

14 in defining the QM segment and MM segment in the system and also choose a cost effect 15 (but also accurate) QM model and a suitable MM force field for treating classical segment.

16 The choice of software program is also a key, as using the one that scales well could be 17 helpful to run the $\mathrm{QM} / \mathrm{MM}$ simulations for large timescales. Such rigorous hybrid 18 simulations can offer extra-ordinary insights about the reaction mechanisms involved 19 between the selected hit(s) and the target(s). Understanding the reaction mechanisms can 20 be useful towards achieving a better lead compound(s). Those leads showing promising in 21 vitro and in vivo activities can be taken to further lead optimization and lengthy and 22 expensive clinical trial stages. Indeed, off-target interactions of drug is yet another 23 important challenge facing the community; and computational methods are also helpful to 24 address this challenge, which is not discussed in this review.

26 The potentials of combining all the computational methods discussed in this review can be 27 best demonstrated, for instance, by a series of studies[86,165-169] carried out on a bacterial 28 enzyme, namely bacterial MurD ligase. A team of scientists from the National Institute of 29 Chemistry, Slovenia, along with their collaborators, have carried out a number of studies 30 on the enzyme. This includes, studying the domain flexibility using MD simulations 31 followed by drug design efforts[165-167], post-docking refinements of the complexes 
1 using MD approaches[167,170], understanding the reaction mechanism(s) of the identified 2 hit-enzyme complexes using QM/MM methods[169] and free energy calculations to 3 understand the binding of inhibitors to the MurD ligase and further drive the design 4 processes[86,168]. In one of the preliminary studies[165], the authors performed extensive 5 targeted MD (TMD) simulations in order to gain some insights into substrate binding 6 process and also the structural changes in the enzyme during the transition(s) between the 7 experimentally determined closed and open states[165]. In another study[166], the authors 8 used this information to perform off-path simulation to obtain a relative energy comparison 9 pathway of the two TMD-generated closing pathways. This study also discerned the 10 pathway which had high-energy demands to perform the biochemical processes[166]. The

11 authors claim that the results from their studies agreed well against the experimental 12 findings[166]. Subsequently, the authors selected three MurD ligase conformations from 13 their MD simulations and used them for two-stage docking-based virtual screening 14 study[167]. The screening identified a panel of promising hits, out of which one of them 15 (an aminothiazole class inhibitor) was confirmed experimentally to act against dual targets, 16 MurD and MurC. The authors re-docked this inhibitor against all the target structures and 17 performed extended classical MD simulations to gain atomistic insights into the ligand18 target interactions [167]. The authors also identified another inhibitor class of benzene-1,319 dicarboxylic acid 2,5-dimethylpyrrole derivatives that showed dual MurD/MurE inhibition 20 properties[170]. In the follow-up study, the authors performed extended MD simulations 21 of this inhibitor-MurD complex to explore their geometrical behaviours. Later, they also 22 performed binding free energy calculations using liner interaction energy (LIE) method 23 that described the energetic behaviour and binding affinity of the compound[170]. Using 24 the information gathered from these studies, the authors again developed new 25 pharmacophore models and performed new phase of virtual screening to only discover 26 novel set of compounds that showed promising effects in the experiments[170]. Similar 27 combination of MD and LIE-based binding free energy calculations were also carried out 28 for Furan-based benzene mono- and dicarboxylic acid derivatives against the bacterial Mur 29 ligases[86]. In their ongoing computational and experimental efforts to design drugs for 30 Mur ligases, the authors also performed advanced QM/MM simulations[169], using 31 B3LYP level of QM theory and CHARMM MM force fields, of the experimental structure 
1 of MurD in the PDB (code: 2UAG). This QM/MM study[169] was useful to understand

2 about the tetrahedral intermediate formation in the enzyme complex, which was not known

3 until then[169]. Hence, the bundle of studies by these authors demonstrate how a series of

4 computational studies (along with experiments) can be set up to advance our knowledge

5 about the structure-properties of a specific target and make progress towards achieving the

6 goal(s) of drug discovery.

\section{Concluding remarks}

11 It has been 38 years since the first molecular dynamics (MD) simulations of bovine 12 pancreatic trypsin inhibitor were carried out for 9.2 picoseconds. Since then, there has been 13 tremendous growth in supercomputing power and significant developments in the accuracy 14 and efficiency of MD-based computational methods. And MD is now well established as 15 an important contributor to drug design and development. With current capacities, MD 16 simulations can be employed for larger biological systems and for microsecond timescales.

17 Such longer classical MD simulations help in effective treatments of the induced-fit effects 18 of the drug binding onto receptors, and can be used to realize optimal drug-receptor binding 19 modes and collect larger conformational samples of the complexes that allow more 20 accurate binding free energy estimations. Alternate versions of classical MD methods, such 21 as CpHMD and enhanced sampling MD approaches, allow tracing chemical changes and 22 other intricate biological events, which normally occur within ligand-protein complexes 23 but are not observed within the practical limits of classical MD simulations. On the other 24 hand, advanced hybrid QM/MM MD methods are useful in revealing the actual reaction 25 mechanisms occurring at the ligand-binding site of the receptor, which are important to 26 design potent ligands that could trigger effective inhibition of the disease targets. Thus MD 27 approaches offer wide range of opportunities and capabilities. Assembling them 28 appropriately with other in silico approaches and experiments can enhance the possibilities 29 of identifying more credible hits that can eventually become effective next-generation 30 drugs to serve human population. 
1 Table 1: Some of the recent studies that employed MD simulations for various applications, such as accounting protein

2 flexibility, post-docking structure refinement and binding free energy calculations, on different targets.

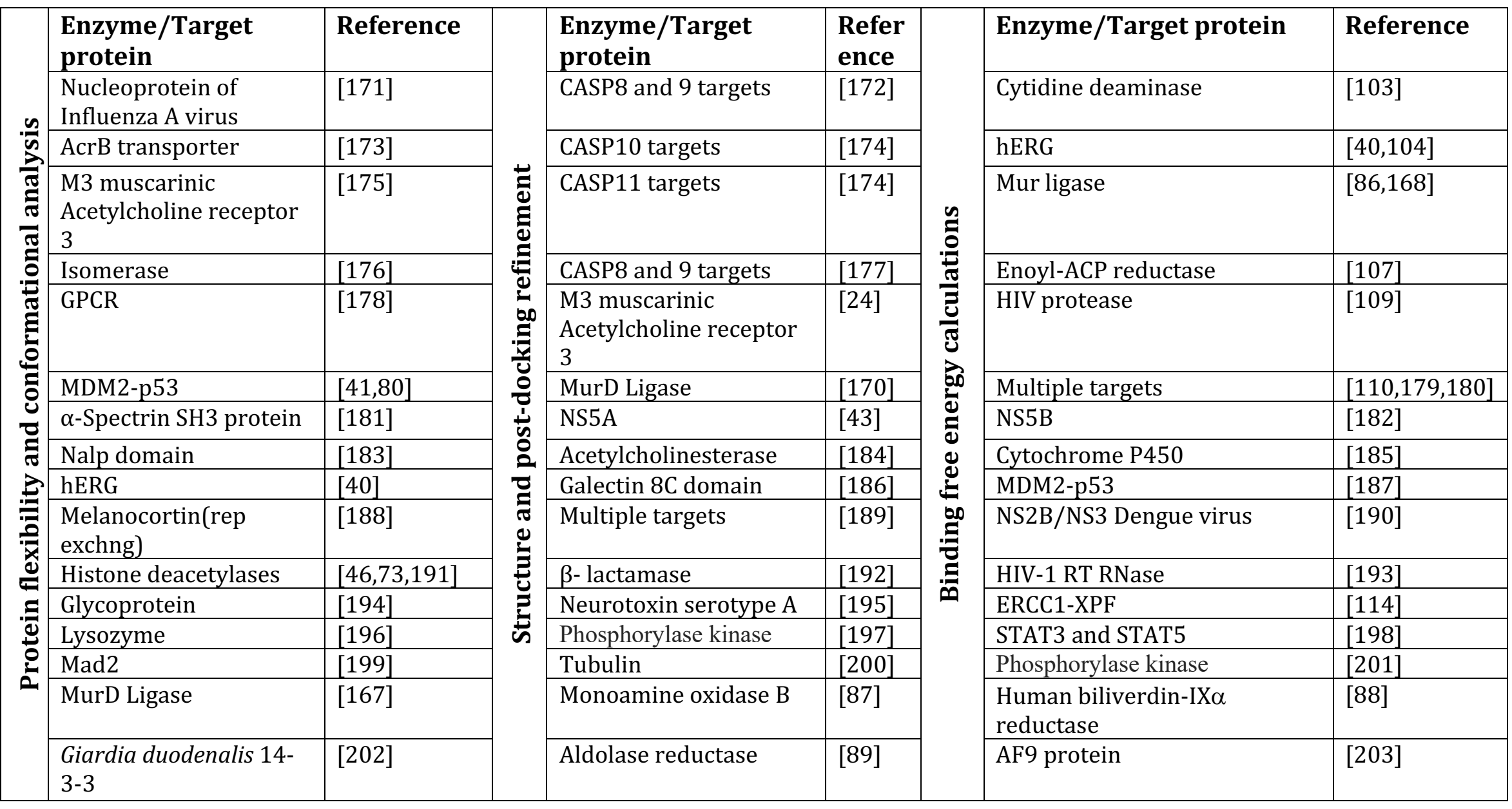


2 Figure captions:

4 Fig. 1: A schematic representation of induced effects of ligand binding to receptor.

5 Fig. 2: Different categories of methods employed for accounting ligand and receptor 6 flexibilities in molecular docking.

7 Fig. 3: Different X-ray crystal structures of histone deacetylases 8 (HDAC 8) showing

8 different conformation of binding site pockets. An overlap of the structures is also shown

9 as a ribbon structure.

10 Fig. 4: Ensembles of structures sampled from long MD trajectories. Twenty-eight

11 structures of MDM2 protein sampled from $50 \mathrm{~ns}$ long MD trajectory (a) and forty-five

12 structures of hERG ion channel captured from 500 ns long MD simulation showing

13 flexibilities of backbone region (b) and side-chain dynamics (c). In (a), the structures of

14 holo-, and apo- trajectories of MDM2 protein are shown in green and blue, respectively.

15 Wherein (b) and (c), the colors indicate flexibility of the concerned segments in the

16 dominant conformations. The representative conformation of the target is shown in Red

17 and the other conformations in the clusters are provided in colours ranging between red to 18 blue.

19 Fig. 5: Binding mode of Daclatasvir with the NS5A protein[43]. The bound drug is shown 20 as a green-colored stick representation and the protein residues are displayed as white sticks

21 (a). The binding sites within the NS5A receptor is also provided as a surface representation 22 (b). 
1 Fig. 6: A QM//MM model of human acetylcholinesterase where residues that can be treated

2 under QM is shown as ball-and-stick and the rest of the systems shown as surface

3 representation and cartoon can be treated with MM force field.

$4 \quad$ Fig. 7: A simplified and practical workflow for molecular modelling and drug design. This

5 work flow lists a sequence of steps that provides an overview of how MD approaches can

6 be stacked along with other modelling and experimental procedures during the drug design

7 and discovery efforts. In addition, a number of key decisions that needs to be taken during

8 each of the modelling and MD stages are also listed. It is important to insist that this work

9 flow in no-way tries to underestimate the roles of experiments in drug discovery. Rather it

10 only tries to highlight the roles of computational approaches, as discussing experimental

11 techniques in detail is not within the scope of present review. 


\section{References}

21 Jeffery, T. and David, M.M. (2006) 1918 Influenza: the Mother of All Pandemics.

3

4

2 Clinton, T.R. et al. (2015) Design and characterization of ebolavirus GP prehairpin intermediate mimics as drug targets. Protein Science 24 (4), 446-463

3 Myers, S. and Baker, A. (2001) Drug discovery[mdash]an operating model for a new era. Nat Biotech 19 (8), 727-730

4 Feher, M. and Schmidt, J.M. (2003) Property Distributions: Differences between Drugs, Natural Products, and Molecules from Combinatorial Chemistry. Journal of Chemical Information and Computer Sciences 43 (1), 218227

$5 \quad$ Lahana, R. (1999) How many leads from HTS? Drug Discovery Today 4 (10), 447-448

6 Newman, D.J. and Cragg, G.M. (2016) Natural Products as Sources of New Drugs from 1981 to 2014. Journal of Natural Products 79 (3), 629-661

7 Schreiber, S.L. (2000) Target-Oriented and Diversity-Oriented Organic Synthesis in Drug Discovery. Science 287 (5460), 1964

8 Luo, T. and Schreiber, S.L. (2009) Gold(I)-Catalyzed Coupling Reactions for the Synthesis of Diverse Small Molecules Using the Build/Couple/Pair Strategy. Journal of the American Chemical Society 131 (15), 5667-5674

9 Kodadek, T. (2011) The rise, fall and reinvention of combinatorial chemistry. Chemical Communications 47 (35), 9757-9763

10 Dorn, M. et al. (2014) Three-dimensional protein structure prediction: Methods and computational strategies. Computational Biology and Chemistry 53, Part B, 251-276

11 Irwin, J.J. and Shoichet, B.K. (2005) ZINC - A Free Database of Commercially Available Compounds for Virtual Screening. Journal of Chemical Information and Modeling 45 (1), 177-182

12 Sterling, T. and Irwin, J.J. (2015) ZINC 15 - Ligand Discovery for Everyone. Journal of Chemical Information and Modeling 55 (11), 2324-2337

13 Pence, H.E. and Williams, A. (2010) ChemSpider: An Online Chemical Information Resource. Journal of Chemical Education 87 (11), 1123-1124

14 Gaulton, A. et al. (2012) ChEMBL: a large-scale bioactivity database for drug discovery. Nucleic Acids Research 40 (Database issue), D1100-D1107

15 Wishart, D.S. et al. (2006) DrugBank: a comprehensive resource for in silico drug discovery and exploration. Nucleic Acids Research 34 (suppl 1), D668D672

16 Bolton, E.E. et al. (2008) Chapter 12 - PubChem: Integrated Platform of Small Molecules and Biological Activities. In Annual Reports in Computational Chemistry (Vol. Volume 4) (Ralph, A.W. and David, C.S., eds.), pp. 217-241, Elsevier

17 Glaab, E. (2015) Building a virtual ligand screening pipeline using free software: a survey. Briefings in Bioinformatics 
118 Alonso, $H$. et al. (2006) Combining docking and molecular dynamic

simulations in drug design. Medicinal Research Reviews 26 (5), 531-568

19 Durrant, J.D. and McCammon, J.A. (2011) Molecular dynamics simulations and drug discovery. BMC Biology 9 (1), 1-9

20 Kerrigan, J. (2013) Molecular Dynamics Simulations in Drug Design. In In Silico Models for Drug Discovery (Vol. 993) (Kortagere, S., ed.), pp. 95-113, Humana Press

21 Zhang, J.-L. et al. (2013) Drug Design Benefits from Molecular Dynamics: Some Examples. Current Computer - Aided Drug Design 9 (4), 532-546

22 Nair, P. and Miners, J. (2014) Molecular dynamics simulations: from structure function relationships to drug discovery. In Silico Pharmacology 2 (1), 1-4

23 Mortier, J. et al. (2015) The impact of molecular dynamics on drug design: applications for the characterization of ligand-macromolecule complexes. Drug Discovery Today 20 (6), 686-702

24 Tautermann, C.S. et al. (2015) What can we learn from molecular dynamics simulations for GPCR drug design? Computational and Structural Biotechnology Journal 13, 111-121

25 Zhao, H. and Caflisch, A. (2015) Molecular dynamics in drug design. European Journal of Medicinal Chemistry 91, 4-14

26 Borhani, D.W. and Shaw, D.E. (2011) The future of molecular dynamics simulations in drug discovery. Journal of Computer-Aided Molecular Design 26 (1), 15-26

27 Huang, S.-Y. et al. (2010) Scoring functions and their evaluation methods for protein-ligand docking: recent advances and future directions. Physical Chemistry Chemical Physics 12 (40), 12899-12908

28 Lang, P.T. et al. (2009) DOCK 6: Combining techniques to model RNA-small molecule complexes. RNA 15 (6), 1219-1230

29 Morris, G.M. et al. (2009) AutoDock4 and AutoDockTools4: Automated docking with selective receptor flexibility. Journal of Computational Chemistry 30 (16), 2785-2791

30 Jones, G. et al. (1997) Development and validation of a genetic algorithm for flexible docking1. Journal of Molecular Biology 267 (3), 727-748

31 Friesner, R.A. et al. (2004) Glide: A New Approach for Rapid, Accurate Docking and Scoring. 1. Method and Assessment of Docking Accuracy. Journal of Medicinal Chemistry 47 (7), 1739-1749

32 Kastritis, P.L. and Bonvin, A.M.J.J. (2012) On the binding affinity of macromolecular interactions: daring to ask why proteins interact. Journal of The Royal Society Interface 10 (79)

33 Koshland, D.E. (1995) The Key-Lock Theory and the Induced Fit Theory. Angewandte Chemie International Edition in English 33 (23-24), 2375-2378

34 Huang, S.-Y. and Zou, X. (2010) Advances and Challenges in Protein-Ligand Docking. International Journal of Molecular Sciences 11 (8), 3016

35 Böhm, H.-J. (1992) The computer program LUDI: A new method for the de novo design of enzyme inhibitors. Journal of Computer-Aided Molecular Design $6(1), 61-78$ 
36 Ferrari, A.M. et al. (2004) Soft Docking and Multiple Receptor Conformations in Virtual Screening. Journal of Medicinal Chemistry 47 (21), 5076-5084

37 Knegtel, R.M.A. et al. (1997) Molecular docking to ensembles of protein structures1. Journal of Molecular Biology 266 (2), 424-440

38 Claußen, H. et al. (2001) FlexE: efficient molecular docking considering protein structure variations1. Journal of Molecular Biology 308 (2), 377-395

39 Fischer, M. et al. (2014) Incorporation of protein flexibility and conformational energy penalties in docking screens to improve ligand discovery. Nat Chem 6 (7), 575-583

40 Anwar-Mohamed, A. et al. (2014) A human ether-á-go-go-related (hERG) ion channel atomistic model generated by long supercomputer molecular dynamics simulations and its use in predicting drug cardiotoxicity. Toxicology Letters 230 (3), 382-392

41 Barakat, K. et al. (2010) Ensemble-based virtual screening reveals dualinhibitors for the p53-MDM2/MDMX interactions. Journal of Molecular Graphics and Modelling 28 (6), 555-568

42 Barakat, K. and Tuszynski, J. (2011) Relaxed complex scheme suggests novel inhibitors for the lyase activity of DNA polymerase beta. Journal of Molecular Graphics and Modelling 29 (5), 702-716

43 Barakat, K.H. et al. (2015) A Refined Model of the HCV NS5A Protein Bound to Daclatasvir Explains Drug-Resistant Mutations and Activity against Divergent Genotypes. Journal of Chemical Information and Modeling 55 (2), 362-373

44 Campbell, A.J. et al. (2014) Ensemble-Based Docking Using Biased Molecular Dynamics. Journal of Chemical Information and Modeling 54 (7), 2127-2138

45 Ge, H. et al. (2013) Molecular Dynamics-Based Virtual Screening: Accelerating the Drug Discovery Process by High-Performance Computing. Journal of Chemical Information and Modeling 53 (10), 2757-2764

46 Kalyaanamoorthy, S. and Chen, Y.-P.P. (2014) Modelling and enhanced molecular dynamics to steer structure-based drug discovery. Progress in Biophysics and Molecular Biology 114 (3), 123-136

47 Mackerell, A.D. (2004) Empirical force fields for biological macromolecules: Overview and issues. Journal of Computational Chemistry 25 (13), 1584-1604

48 Jones, J.E. (1924) On the Determination of Molecular Fields. II. From the Equation of State of a Gas. Proceedings of the Royal Society of London A: Mathematical, Physical and Engineering Sciences 106 (738), 463-477

49 Darden, T. et al. (1993) Particle mesh Ewald: An $N \cdot \log (\mathrm{N})$ method for Ewald sums in large systems. The Journal of Chemical Physics 98 (12), 10089-10092

50 MacKerell Jr, A.D. et al. (1998) All-atom empirical potential for molecular modeling and dynamics studies of proteins. Journal of Physical Chemistry B 102 (18), 3586-3616

51 Cornell, W.D. et al. (1995) A second generation force field for the simulation of proteins, nucleic acids, and organic molecules. Journal of the American Chemical Society 117 (19), 5179-5197

52 Daura, X. et al. (1998) Parametrization of aliphatic CHn united atoms of GROMOS96 force field. Journal of Computational Chemistry 19 (5), 535-547 
53 González, M.A. (2011) Force fields and molecular dynamics simulations. JDN $12,169-200$

54 Todorova, N. et al. (2008) Systematic Comparison of Empirical Forcefields for Molecular Dynamic Simulation of Insulin. The Journal of Physical Chemistry B 112 (35), 11137-11146

55 Nguyen, T.T. et al. (2014) Effects of Water Models on Binding Affinity: Evidence from All-Atom Simulation of Binding of Tamiflu to A/H5N1 Neuraminidase. The Scientific World Journal 2014, 536084

56 Becker, C.A. et al. (2013) Considerations for choosing and using force fields and interatomic potentials in materials science and engineering. Current Opinion in Solid State and Materials Science 17 (6), 277-283

\section{7} Verlet, L. (1967) Computer "experiments" on classical fluids. I. Thermodynamical properties of Lennard-Jones molecules. Physical Review 159 (1), 98-103

58 Swope, W.C. et al. (1982) A computer simulation method for the calculation of equilibrium constants for the formation of physical clusters of molecules: Application to small water clusters. The Journal of Chemical Physics 76 (1), 637-649

59 Cuendet, M.A. and van Gunsteren, W.F. (2007) On the calculation of velocitydependent properties in molecular dynamics simulations using the leapfrog integration algorithm. The Journal of Chemical Physics 127 (18), 184102

60 Ryckaert, J.-P. et al. (1977) Numerical integration of the cartesian equations of motion of a system with constraints: molecular dynamics of n-alkanes. Journal of Computational Physics 23 (3), 327-341

61 Andersen, H.C. (1983) Rattle: A "velocity" version of the shake algorithm for molecular dynamics calculations. Journal of Computational Physics 52 (1), 2434

62 Hess, B. et al. (1997) LINCS: A Linear Constraint Solver for molecular simulations. Journal of Computational Chemistry 18 (12), 1463-1472

63 McQuarrie, D.A. (2000) Statistical Mechanics, University Science Books

64 Beard, D.A. and Qian, H. (2008) Chemical Biophysics: Quantitative Analysis of Cellular Systems, Cambridge University Press

65 Richardson, J.M. and Brinkley Jr, S.R. (1960) Cell method in grand canonical ensemble. The Journal of Chemical Physics 33 (5), 1467-1478

66 Corti, D.S. and Soto-Campos, G. (1998) Deriving the isothermal-isobaric ensemble: The requirement of a "shell" molecule and applicability to small systems. Journal of Chemical Physics 108 (19), 7959-7966

67 Adelman, S.A. and Doll, J.D. (1976) Generalized Langevin equation approach for atom/solid - surface scattering: General formulation for classical scattering off harmonic solids. The Journal of Chemical Physics 64 (6), 23752388

68 Berendsen, H.J.C. et al. (1984) Molecular dynamics with coupling to an external bath. The Journal of Chemical Physics 81 (8), 3684-3690

69 Nosé, S. (1984) A unified formulation of the constant temperature molecular dynamics methods. The Journal of Chemical Physics 81 (1), 511-519 
170 Hoover, W.G. (1985) Canonical dynamics: Equilibrium phase-space

71 Deschamps, N. et al. (2015) How the flexibility of human histone deacetylases influences ligand binding: an overview. Drug Discovery Today 20 (6), 736-742

72 Ma, B. et al. (1999) Folding funnels and binding mechanisms. Protein Engineering 12 (9), 713-720

73 Kalyaanamoorthy, S. and Chen, Y.-P.P. (2014) A steered molecular dynamics mediated hit discovery for histone deacetylases. Physical Chemistry Chemical Physics 16 (8), 3777-3791

74 Estiu, G. et al. (2010) On the inhibition of histone deacetylase 8. Bioorganic \& Medicinal Chemistry 18 (11), 4103-4110

75 Horn, J.R. and Shoichet, B.K. (2004) Allosteric Inhibition Through Core Disruption. Journal of Molecular Biology 336 (5), 1283-1291

76 Diskin, R. et al. (2008) A Novel Lipid Binding Site Formed by the MAP Kinase Insert in p38 $\alpha$. Journal of Molecular Biology 375 (1), 70-79

77 Schames, J.R. et al. (2004) Discovery of a Novel Binding Trench in HIV Integrase. Journal of Medicinal Chemistry 47 (8), 1879-1881

78 Hazuda, D.J. et al. (2004) A naphthyridine carboxamide provides evidence for discordant resistance between mechanistically identical inhibitors of HIV-1 integrase. Proceedings of the National Academy of Sciences of the United States of America 101 (31), 11233-11238

79 David, C. and Jacobs, D. (2014) Principal Component Analysis: A Method for Determining the Essential Dynamics of Proteins. In Protein Dynamics (Vol. 1084) (Livesay, D.R., ed.), pp. 193-226, Humana Press

80 Bowman, A.L. et al. (2007) Small Molecule Inhibitors of the MDM2-p53 Interaction Discovered by Ensemble-Based Receptor Models. Journal of the American Chemical Society 129 (42), 12809-12814

81 Rakers, C. et al. (2015) Computational close up on protein-protein interactions: how to unravel the invisible using molecular dynamics simulations? Wiley Interdisciplinary Reviews: Computational Molecular Science 5 (5), 345-359

82 Sousa, S.F. et al. (2011) Detailed Atomistic Analysis of the HIV-1 Protease Interface. The Journal of Physical Chemistry B 115 (21), 7045-7057

83 Viricel, C. et al. (2015) Human PD-1 binds differently to its human ligands: A comprehensive modeling study. Journal of Molecular Graphics and Modelling $57,131-142$

84 Rastelli, G. et al. (2002) Discovery of new inhibitors of aldose reductase from molecular docking and database screening. Bioorganic \& Medicinal Chemistry 10 (5), 1437-1450

85 Cavalli, A. et al. (2004) A Computational Study of the Binding of Propidium to the Peripheral Anionic Site of Human Acetylcholinesterase. Journal of Medicinal Chemistry 47 (16), 3991-3999

86 Perdih, A. et al. (2015) Furan-based benzene mono- and dicarboxylic acid derivatives as multiple inhibitors of the bacterial Mur ligases (MurC-MurF): experimental and computational characterization. Journal of Computer-Aided Molecular Design 29 (6), 541-560 
187 Distinto, S. et al. (2012) Synthesis and biological assessment of novel 2thiazolylhydrazones and computational analysis of their recognition by monoamine oxidase B. European Journal of Medicinal Chemistry 48, 284-295

$88 \mathrm{Fu}$, G. et al. (2012) Molecular Modeling to Provide Insight into the Substrate Binding and Catalytic Mechanism of Human Biliverdin-IXa Reductase. The Journal of Physical Chemistry B 116 (32), 9580-9594

89 Vijjulatha, M. et al. (2014) Induced fit docking, pharmacophore modeling, and molecular dynamic simulations on thiazolidinedione derivatives to explore key interactions with Tyr48 in polyol pathway. Journal of Molecular Modeling 20 (7), 1-14

90 Huang, Y.-ming M. et al. (2012) Insights from Free-Energy Calculations: Protein Conformational Equilibrium, Driving Forces, and Ligand-Binding Modes. Biophysical Journal 103 (2), 342-351

91 Michel, J. et al. (2010) Rigorous Free Energy Calculations in Structure-Based Drug Design. Molecular Informatics 29 (8-9), 570-578

92 Christ, C.D. and Fox, T. (2014) Accuracy Assessment and Automation of Free Energy Calculations for Drug Design. Journal of Chemical Information and Modeling 54 (1), 108-120

93 Rathore, R.S. et al. (2013) Advances in binding free energies calculations: QM/MM-based free energy perturbation method for drug design. Current Pharmaceutical Design 19 (26), 4674-4686

94 Wereszczynski, J. and McCammon, J.A. (2012) Statistical mechanics and molecular dynamics in evaluating thermodynamic properties of biomolecular recognition. Quarterly Reviews of Biophysics 45 (01), 1-25

95 Camps, P. et al. (2000) New Tacrine-Huperzine A Hybrids (Huprines): Highly Potent Tight-Binding Acetylcholinesterase Inhibitors of Interest for the Treatment of Alzheimer's Disease. Journal of Medicinal Chemistry 43 (24), 4657-4666

96 Rami Reddy, M. et al. (2000) Free energy calculations: Use and limitations in predicting ligand binding affinities. In Reviews in Computational Chemistry (Vol. 16), pp. 217-304

97 Jorgensen, W.L. et al. (2006) Computer-aided design of non-nucleoside inhibitors of HIV-1 reverse transcriptase. Bioorganic \& Medicinal Chemistry Letters 16 (3), 663-667

98 Kim, J.T. et al. (2006) FEP-Guided Selection of Bicyclic Heterocycles in Lead Optimization for Non-Nucleoside Inhibitors of HIV-1 Reverse Transcriptase. Journal of the American Chemical Society 128 (48), 15372-15373

99 Michel, J. et al. (2009) In Silico Improvement of $\beta 3$-Peptide Inhibitors of p53•hDM2 and p53•hDMX. Journal of the American Chemical Society 131 (18), 6356-6357

100 Wang, L. et al. (2015) Accurate and Reliable Prediction of Relative Ligand Binding Potency in Prospective Drug Discovery by Way of a Modern FreeEnergy Calculation Protocol and Force Field. Journal of the American Chemical Society 137 (7), 2695-2703

101 Åqvist, J. et al. (1994) A new method for predicting binding affinity in computer-aided drug design. Protein Engineering 7 (3), 385-391 
102 Nicolotti, O. et al. (2010) Screening of benzamidine-based thrombin inhibitors via a linear interaction energy in continuum electrostatics model. Journal of Computer-Aided Molecular Design 24 (2), 117-129

103 Timmers, L. et al. (2012) Combining molecular dynamics and docking simulations of the cytidine deaminase from Mycobacterium tuberculosis H37Rv. Journal of Molecular Modeling 18 (2), 467-479

104 Boukharta, L. et al. (2011) Computer Simulations of Structure-Activity Relationships for hERG Channel Blockers. Biochemistry 50 (27), 6146-6156

105 Gutiérrez-de-Terán, H. et al. (2006) Computational analysis of plasmepsin IV bound to an allophenylnorstatine inhibitor. FEBS Letters 580 (25), 5910-5916

106 Gutiérrez-de-Terán, H. and Åqvist, J. (2012) Linear Interaction Energy: Method and Applications in Drug Design. In Computational Drug Discovery and Design (Vol. 819) (Baron, R., ed.), pp. 305-323, Springer New York

107 Su, P.-C. et al. (2015) Comparison of radii sets, entropy, QM methods, and sampling on MM-PBSA, MM-GBSA, and QM/MM-GBSA ligand binding energies of F. tularensis enoyl-ACP reductase (FabI). Journal of Computational Chemistry 36 (25), 1859-1873

108 Genheden, S. and Ryde, U. (2015) The MM/PBSA and MM/GBSA methods to estimate ligand-binding affinities. Expert Opinion on Drug Discovery 10 (5), 449-461

109 Oehme, D.P. et al. (2012) Effect of atomic charge, solvation, entropy, and ligand protonation state on MM-PB(GB)SA binding energies of HIV protease. Journal of Computational Chemistry 33 (32), 2566-2580

110 Hou, T. et al. (2011) Assessing the Performance of the MM/PBSA and MM/GBSA Methods. 1. The Accuracy of Binding Free Energy Calculations Based on Molecular Dynamics Simulations. Journal of Chemical Information and Modeling 51 (1), 69-82

111 Sun, H. et al. (2014) Assessing the performance of MM/PBSA and MM/GBSA methods. 5. Improved docking performance using high solute dielectric constant MM/GBSA and MM/PBSA rescoring. Physical Chemistry Chemical Physics 16 (40), 22035-22045

112 Hou, X. et al. (2013) How to Improve Docking Accuracy of AutoDock4.2: A Case Study Using Different Electrostatic Potentials. Journal of Chemical Information and Modeling 53 (1), 188-200

113 Genheden, S. and Ryde, U. (2010) How to obtain statistically converged MM/GBSA results. Journal of Computational Chemistry 31 (4), 837-846

114 Jordheim, L.P. et al. (2013) Small Molecule Inhibitors of ERCC1-XPF ProteinProtein Interaction Synergize Alkylating Agents in Cancer Cells. Molecular Pharmacology 84 (1), 12-24

115 Kalyaanamoorthy, S. and Chen, Y.-P.P. (2013) Quantum polarized ligand docking investigation to understand the significance of protonation states in histone deacetylase inhibitors. Journal of Molecular Graphics and Modelling 44, 44-53

116 Velez-Vega, C. et al. (2015) Estimation of Solvation Entropy and Enthalpy via Analysis of Water Oxygen-Hydrogen Correlations. Journal of Chemical Theory and Computation 11 (11), 5090-5102 
117 Wong, S.E. and Lightstone, F.C. (2011) Accounting for water molecules in drug design. Expert Opinion on Drug Discovery 6 (1), 65-74

118 Riniker, S. et al. (2012) Free enthalpies of replacing water molecules in protein binding pockets. Journal of Computer-Aided Molecular Design 26 (12), 12931309

119 Li, Z. and Lazaridis, T. (2006) Thermodynamics of Buried Water Clusters at a Protein-Ligand Binding Interface. The Journal of Physical Chemistry B 110 (3), 1464-1475

120 Ramsey, S. et al. (2016) Solvation thermodynamic mapping of molecular surfaces in AmberTools: GIST. Journal of Computational Chemistry 37 (21), 2029-2037

121 Young, T. et al. (2007) Motifs for molecular recognition exploiting hydrophobic enclosure in protein-ligand binding. Proceedings of the National Academy of Sciences 104 (3), 808-813

122 Baroni, M. et al. (2007) A Common Reference Framework for Analyzing/Comparing Proteins and Ligands. Fingerprints for Ligands And Proteins (FLAP): Theory and Application. Journal of Chemical Information and Modeling 47 (2), 279-294

123 Bayden, A.S. et al. (2015) Evaluating Free Energies of Binding and Conservation of Crystallographic Waters Using SZMAP. Journal of Chemical Information and Modeling 55 (8), 1552-1565

124 Nguyen, C.N. et al. (2012) Grid inhomogeneous solvation theory: Hydration structure and thermodynamics of the miniature receptor cucurbit[7]uril. The Journal of Chemical Physics 137 (4), 044101

125 Velez-Vega, C. et al. (2014) Time-Averaged Distributions of Solute and Solvent Motions: Exploring Proton Wires of GFP and PfM2DH. Journal of Chemical Information and Modeling 54 (12), 3344-3361

126 Cui, G. et al. (2013) SPAM: A Simple Approach for Profiling Bound Water Molecules. Journal of Chemical Theory and Computation 9 (12), 5539-5549

127 Li, Z. and Lazaridis, T. (2012) Computing the Thermodynamic Contributions of Interfacial Water. In Computational Drug Discovery and Design (Baron, R., ed.), pp. 393-404, Springer New York

128 Beuming, T. et al. (2009) High-energy water sites determine peptide binding affinity and specificity of PDZ domains. Protein Science 18 (8), 1609-1619

129 Beuming, T. et al. (2012) Thermodynamic analysis of water molecules at the surface of proteins and applications to binding site prediction and characterization. Proteins: Structure, Function, and Bioinformatics 80 (3), 871883

130 Abel, R. et al. (2011) Contribution of Explicit Solvent Effects to the Binding Affinity of Small-Molecule Inhibitors in Blood Coagulation Factor Serine Proteases. ChemMedChem 6 (6), 1049-1066

131 Guimarães, C.R.W. and Mathiowetz, A.M. (2010) Addressing Limitations with the MM-GB/SA Scoring Procedure using the WaterMap Method and Free Energy Perturbation Calculations. Journal of Chemical Information and Modeling 50 (4), 547-559 
1132 Higgs, C. et al. (2010) Hydration Site Thermodynamics Explain SARs for Triazolylpurines Analogues Binding to the A2A Receptor. ACS Medicinal Chemistry Letters 1 (4), 160-164

133 Kim, M.O. and McCammon, J.A. (2016) Computation of pH-dependent binding free energies. Biopolymers 105 (1), 43-49

134 Onufriev, A.V. and Alexov, E. (2013) Protonation and pK changes in proteinligand binding. Quarterly Reviews of Biophysics 46 (02), 181-209

135 Wallace, J.A. and Shen, J.K. (2012) Charge-leveling and proper treatment of long-range electrostatics in all-atom molecular dynamics at constant $\mathrm{pH}$. The Journal of Chemical Physics 137 (18), 184105

136 Adcock, S.A. and McCammon, J.A. (2006) Molecular Dynamics: Survey of Methods for Simulating the Activity of Proteins. Chemical Reviews 106 (5), 1589-1615

137 Donnini, S. et al. (2011) Constant pH Molecular Dynamics in Explicit Solvent with $\lambda$-Dynamics. Journal of Chemical Theory and Computation 7 (6), 19621978

138 Lee, J. et al. (2016) Computational scheme for pH-dependent binding free energy calculation with explicit solvent. Protein Science 25 (1), 231-243

139 Wallace, J.A. and Shen, J.K. (2011) Continuous Constant pH Molecular Dynamics in Explicit Solvent with pH-Based Replica Exchange. Journal of Chemical Theory and Computation 7 (8), 2617-2629

140 Swails, J.M. and Roitberg, A.E. (2012) Enhancing Conformation and Protonation State Sampling of Hen Egg White Lysozyme Using pH Replica Exchange Molecular Dynamics. Journal of Chemical Theory and Computation 8 (11), 4393-4404

141 Lee, J. et al. (2014) Constant pH Molecular Dynamics in Explicit Solvent with Enveloping Distribution Sampling and Hamiltonian Exchange. Journal of Chemical Theory and Computation 10 (7), 2738-2750

142 Meller, J.a. (2001) Molecular Dynamics. In $e L S$, John Wiley \& Sons, Ltd

143 Onuchic, J.N. et al. (1997) Theory of Protein Folding: The Energy Landscape Perspective. In Annual Review of Physical Chemistry (Vol. 48), pp. 545-600

144 Marsili, S. et al. (2010) ORAC: A molecular dynamics simulation program to explore free energy surfaces in biomolecular systems at the atomistic level. Journal of Computational Chemistry 31 (5), 1106-1116

145 Bernardi, R.C. et al. (2015) Enhanced sampling techniques in molecular dynamics simulations of biological systems. Biochimica et Biophysica Acta (BBA) - General Subjects 1850 (5), 872-877

146 De Vivo, M. et al. (2016) Role of Molecular Dynamics and Related Methods in Drug Discovery. Journal of Medicinal Chemistry

147 Rodriguez-Bussey, I.G. et al. (2016) Enhanced molecular dynamics sampling of drug target conformations. Biopolymers 105 (1), 35-42

148 Saunders, M.G. and Voth, G.A. (2013) Coarse-Graining Methods for Computational Biology. Annual Review of Biophysics 42 (1), 73-93

149 Senftle, T.P. et al. (2016) The ReaxFF reactive force-field: development, applications and future directions. Npj Computational Materials 2, 15011 
1150 Han, Y. et al. (2016) Development, applications and challenges of ReaxFF reactive force field in molecular simulations. Frontiers of Chemical Science and Engineering 10 (1), 16-38

151 Baker, C.M. (2015) Polarizable force fields for molecular dynamics simulations of biomolecules. Wiley Interdisciplinary Reviews: Computational Molecular Science 5 (2), 241-254

152 Ren, P. and Ponder, J.W. (2003) Polarizable Atomic Multipole Water Model for Molecular Mechanics Simulation. The Journal of Physical Chemistry B 107 (24), 5933-5947

153 Ponder, J.W. et al. (2010) Current Status of the AMOEBA Polarizable Force Field. The Journal of Physical Chemistry B 114 (8), 2549-2564

154 Tuckerman, M.E. (2002) Ab initio molecular dynamics: basic concepts, current trends and novel applications Journal of Physics: Condensed Matter 14

155 Car, R. and Parrinello, M. (1985) Unified Approach for Molecular Dynamics and Density-Functional Theory. Physical Review Letters 55 (22), 2471-2474

156 Lorenz, C. and Doltsinis, N.L. (2012) Molecular Dynamics Simulation: From "Ab Initio" to "Coarse Grained". In Handbook of Computational Chemistry, pp. 195-238, Springer

157 Warshel, A. and Levitt, M. (1976) Theoretical studies of enzymic reactions: Dielectric, electrostatic and steric stabilization of the carbonium ion in the reaction of lysozyme. Journal of Molecular Biology 103 (2), 227-249

158 Laio, A. et al. (2002) A Hamiltonian electrostatic coupling scheme for hybrid Car-Parrinello molecular dynamics simulations. The Journal of Chemical Physics 116 (16), 6941-6947

159 Svensson, M. et al. (1996) ONIOM: A Multilayered Integrated MO + MM Method for Geometry Optimizations and Single Point Energy Predictions. A Test for Diels-Alder Reactions and $\mathrm{Pt}(\mathrm{P}(\mathrm{t}-\mathrm{Bu}) 3) 2+\mathrm{H} 2$ Oxidative Addition. The Journal of Physical Chemistry 100 (50), 19357-19363

160 Vreven, T. et al. (2006) Combining Quantum Mechanics Methods with Molecular Mechanics Methods in ONIOM. Journal of Chemical Theory and Computation 2 (3), 815-826

161 Chen, J. et al. (2015) A comparative study of trypsin specificity based on QM/MM molecular dynamics simulation and QM/MM GBSA calculation. Journal of Biomolecular Structure and Dynamics, 1-13

162 Wu, E.L. et al. (2009) Determination of the Structure Form of the Fourth Ligand of Zinc in Acutolysin A Using Combined Quantum Mechanical and Molecular Mechanical Simulation. The Journal of Physical Chemistry B 113 (8), 2477-2485

163 Riccardi, D. et al. (2005) pKa Calculations in Solution and Proteins with QM/MM Free Energy Perturbation Simulations: A Quantitative Test of QM/MM Protocols. The Journal of Physical Chemistry B 109 (37), 17715-17733

164 Grotendorst, J. and Simulation, I.f.A. (2012) Hierarchical Methods for Dynamics in Complex Molecular Systems, Forschungszentrum, Zentralbibliothek

165 Perdih, A. et al. (2007) Targeted molecular dynamics simulation studies of binding and conformational changes in E. coli MurD. Proteins: Structure, Function, and Bioinformatics 68 (1), 243-254 
166 Perdih, A. and Solmajer, T. (2012) MurD ligase from Escherichia coli: Cterminal domain closing motion. Computational and Theoretical Chemistry 979 (Complete), 73-81

167 Perdih, A. et al. (2014) Inhibitor Design Strategy Based on an Enzyme Structural Flexibility: A Case of Bacterial MurD Ligase. Journal of Chemical Information and Modeling 54 (5), 1451-1466

168 Perdih, A. et al. (2013) Molecular dynamics simulation and linear interaction energy study of d-Glu-based inhibitors of the MurD ligase. Journal of ComputerAided Molecular Design 27 (8), 723-738

169 Perdih, A. et al. (2009) MurD ligase from E. coli: Tetrahedral intermediate formation study by hybrid quantum mechanical/molecular mechanical replica path method. Proteins: Structure, Function, and Bioinformatics 74 (3), 744-759

170 Perdih, A. et al. (2014) Benzene-1,3-dicarboxylic acid 2,5-dimethylpyrrole derivatives as multiple inhibitors of bacterial Mur ligases (MurC-MurF). Bioorganic \& Medicinal Chemistry 22 (15), 4124-4134

171 Tarus, B. et al. (2012) Molecular Dynamics Studies of the Nucleoprotein of Influenza A Virus: Role of the Protein Flexibility in RNA Binding. PLoS ONE 7 (1), e30038

172 Mirjalili, V. et al. (2014) Physics-based protein structure refinement through multiple molecular dynamics trajectories and structure averaging. Proteins: Structure, Function, and Bioinformatics 82, 196-207

173 Vargiu, A.V. and Nikaido, H. (2012) Multidrug binding properties of the AcrB efflux pump characterized by molecular dynamics simulations. Proceedings of the National Academy of Sciences 109 (50), 20637-20642

174 Feig, M. (2016) Local Protein Structure Refinement via Molecular Dynamics Simulations with locPREFMD. Journal of Chemical Information and Modeling 56 (7), 1304-1312

175 Kruse, A.C. et al. (2012) Structure and dynamics of the M3 muscarinic acetylcholine receptor. Nature 482 (7386), 552-556

176 Römer, R.A. et al. (2016) The flexibility and dynamics of protein disulphideisomerase. Proteins: Structure, Function, and Bioinformatics, n/a-n/a

177 Mirjalili, V. and Feig, M. (2013) Protein Structure Refinement through Structure Selection and Averaging from Molecular Dynamics Ensembles. Journal of Chemical Theory and Computation 9 (2), 1294-1303

178 Preininger, A.M. et al. (2013) Conformational Flexibility and Structural Dynamics in GPCR-Mediated G Protein Activation: A Perspective. Journal of Molecular Biology 425 (13), 2288-2298

179 Sun, H. et al. (2014) Assessing the performance of MM/PBSA and MM/GBSA methods. 4. Accuracies of MM/PBSA and MM/GBSA methodologies evaluated by various simulation protocols using PDBbind data set. Physical Chemistry Chemical Physics 16 (31), 16719-16729

180 Sgobba, M. et al. (2012) Application of a post-docking procedure based on MMPBSA and MM-GBSA on single and multiple protein conformations. European Journal of Medicinal Chemistry 58, 431-440 
1181 Fraccalvieri, D. et al. (2011) Conformational and functional analysis of molecular dynamics trajectories by Self-Organising Maps. BMC Bioinformatics $12(1), 1-19$

182 Pan, D. et al. (2016) Computational study on the drug resistance mechanism of hepatitis C virus NS5B RNA-dependent RNA polymerase mutants to BMS791325 by molecular dynamics simulation and binding free energy calculations. Chemometrics and Intelligent Laboratory Systems 154, 185-193

183 Sahoo, B.R. et al. (2014) A conformational analysis of mouse Nalp3 domain structures by molecular dynamics simulations, and binding site analysis. Molecular BioSystems 10 (5), 1104-1116

184 Fang, J. et al. (2014) Inhibition of acetylcholinesterase by two genistein derivatives: kinetic analysis, molecular docking and molecular dynamics simulation. Acta Pharmaceutica Sinica B 4 (6), 430-437

$185 \mathrm{Lu}, \mathrm{H}$. et al. (2014) Binding free energies for nicotine analogs inhibiting cytochrome P450 $2 \mathrm{~A} 6$ by a combined use of molecular dynamics simulations and QM/MM-PBSA calculations. Bioorganic \& Medicinal Chemistry 22 (7), 2149-2156

186 Kumar, S. et al. (2013) Understanding the Specificity of Human Galectin-8C Domain Interactions with Its Glycan Ligands Based on Molecular Dynamics Simulations. PLoS ONE 8 (3), e59761

187 Niu, R.-J. et al. (2013) Molecular dynamics simulations studies and free energy analysis on inhibitors of MDM2-p53 interaction. Journal of Molecular Graphics and Modelling 46, 132-139

188 Shahlaei, M. and Mousavi, A. (2015) A Conformational Analysis Study on the Melanocortin 4 Receptor Using Multiple Molecular Dynamics Simulations. Chemical Biology \& Drug Design 86 (3), 309-321

189 Proctor, Elizabeth A. et al. (2012) Discrete Molecular Dynamics Distinguishes Nativelike Binding Poses from Decoys in Difficult Targets. Biophysical Journal $102(1), 144-151$

190 Mukhametov, A. et al. (2014) Allosteric pocket of the dengue virus (serotype 2) NS2B/NS3 protease: In silico ligand screening and molecular dynamics studies of inhibition. Journal of Molecular Graphics and Modelling 52, 103-113

191 Kalyaanamoorthy, S. and Chen, Y.-P.P. (2013) Ligand release mechanisms and channels in histone deacetylases. Journal of Computational Chemistry 34 (26), 2270-2283

192 Kumar, K.M. et al. (2014) Molecular docking and molecular dynamics studies on [small beta]-lactamases and penicillin binding proteins. Molecular BioSystems 10 (4), 891-900

193 Poongavanam, V. et al. (2014) Binding free energy based structural dynamics analysis of HIV-1 RT RNase H-inhibitor complexes. Integrative Biology 6 (11), 1010-1022

194 Wen, P.-C. et al. (2013) On the Origin of Large Flexibility of P-glycoprotein in the Inward-facing State. Journal of Biological Chemistry 288 (26), 1921119220 
$195 \mathrm{Hu}, \mathrm{X}$. et al. (2014) Structural insight into exosite binding and discovery of novel exosite inhibitors of botulinum neurotoxin serotype A through in silico screening. Journal of Computer-Aided Molecular Design 28 (7), 765-778

196 Wang, K. et al. (2015) Hierarchical Conformational Analysis of Native Lysozyme Based on Sub-Millisecond Molecular Dynamics Simulations. PLoS ONE 10 (6), e0129846

197 Hayes, J.M. et al. (2011) Kinetics, in silico docking, molecular dynamics, and MM-GBSA binding studies on prototype indirubins, KT5720, and staurosporine as phosphorylase kinase ATP-binding site inhibitors: The role of water molecules examined. Proteins: Structure, Function, and Bioinformatics 79 (3), 703-719

198 Shao, S. et al. (2014) Dual-inhibitors of STAT5 and STAT3: studies from molecular docking and molecular dynamics simulations. Journal of Molecular Modeling 20 (8), 1-17

$199 \mathrm{Li}, \mathrm{C}$. et al. (2014) Molecular Dynamics Simulation on the Conformational Transition of the Mad2 Protein from the Open to the Closed State. International Journal of Molecular Sciences 15 (4)

200 Yadava, U. et al. (2015) Stabilization of Microtubules by Taxane Diterpenoids: Insight from Docking and MD simulations. Journal of Biological Physics 41 (2), 117-133

201 Begum, J. et al. (2015) An evaluation of indirubin analogues as phosphorylase kinase inhibitors. Journal of Molecular Graphics and Modelling 61, 231-242

202 Cau, Y. et al. (2015) Molecular Dynamics Simulations and Structural Analysis of Giardia duodenalis 14-3-3 Protein-Protein Interactions. Journal of Chemical Information and Modeling 55 (12), 2611-2622

203 Wang, Q. et al. (2016) Exploring the mechanism how AF9 recognizes and binds H3K9ac by molecular dynamics simulations and free energy calculations. Biopolymers 105 (11), 779-786 


\section{BOX 1: Important quantities in MD analyses}

\section{Root mean square deviation (RMSD)}

RMSD is a measure of the average deviation or distance between the atoms when threedimensional structures are superimposed on each other. When analysing an MD trajectory, this value (or RMSD) could be a very important quantity that is useful to trace how much the structure that underwent MD simulations has deviated from its' starting structure.

\section{Interaction energy}

The interaction energy is the amount of energy that is caused by the interaction(s) between two residues (or objects) and its' contribution towards the total energy of the system. Interaction energies between different amino acid residues from the target and the bound ligand could make significant impact on the binding affinity of the complex. Thus, identifying the key residues that possess high interaction energy against the ligand is important in binding mode analyses.

\section{Interaction distance}

It is a minimum distance between two non-bonded residues of proteins or between residues and ligand that could affect each other, thereby impacting the total energy of the system.

\section{Correlation functions}

They are mathematical descriptors that connect the properties of protein structures with that of their significance. Thus correlation function remains an important tool for protein structure analyses from the MD trajectories.

\section{Radial distribution function (RDF)}

RDF is a quantity that describes the average radial packaging of atoms in a system and can be calculated by constructing normalized histograms of atom pair distances with respect to an ideal gas.

$$
g(r)=\frac{n(r)}{4 \pi r^{2} \rho \Delta r}
$$

Where, $n(r)$ is the number of atoms in a shell of width $\Delta r$ at distance $r$ and $\rho$ is the mean atom density. This quantity can be useful, for instance, to identify how many waters are coordinating with a metal ion in the active site of the protein during the course of MD simulation.

\section{Hydrogen bond (H-bond)}

The electrostatic force that attracts the hydrogen attached to one electronegative atom to another electronegative atom holding lone pair of electrons. Thus, identifying the number of $\mathrm{H}-$ bonds between the bound ligand and its' surrounding amino acid residues of the protein is one of the key step while analysing the MD trajectories. 


\section{BOX 2: Glossary of terms}

\section{Virtual screening}

Virtual screening (or in silico screening) is a computational approach employed in structure-based drug design to screen a library (or libraries) of small-molecules against the desired protein target in order to rank them based on their affinities to the concerned binding site of the target.

\section{Molecular docking}

A method to predict the favoured binding orientations between two molecules to form a stable complex

\section{Scoring function}

Mathematical method to quantify the interactions between two molecules when they are docked together.

\section{Shape matching}

A sampling method that uses receptor-complementarity as a criterion for identifying the ligand binding conformations

\section{Stochastic algorithms}

A sampling method that incorporates random changes to the ligand in transitional, rotational and conformational space to identify the most suitable ligand binding conformation

\section{Systematic search}

A sampling method that utilizes all degrees of freedom to sample the ligand binding conformations

\section{Induced-fit effect}

Conformational changes in an enzyme triggered by the interactions with (or binding of) small molecules or other proteins.

\section{Periodic boundary condition (PBC)}

Periodic boundary condition (or PBC) is a method employed in MD simulations to eliminate the issues concerning boundary effects, arising from finite size, by treating the system as infinite with the help of a unit cell.

\section{Free energy of binding}

Within the context of ligand-protein complex in drug design, the free energy of binding is defined as the free energy difference between the ligand-bound state (complex) and the free unbound states (free protein and free ligand). 
BOX 3: List of abbreviations

CADD: Computer-aided drug design

SBDD: Structure-based drug design

MD: Molecular dynamics

PDB: Protein data bank

QM/MM: Quantum Mechanics/Molecular Mechanics

IFD: Induced-fit docking

FEP: Free energy perturbation

LIE: Liner Interaction Energy

TI: Thermodynamics Integration

MM-GBSA: Molecular Mechanics-generalized Born surface area

MM-PBSA: Molecular Mechanics-Poisson-Boltzmann surface area 
UniProtKB/

Swiss-Prot
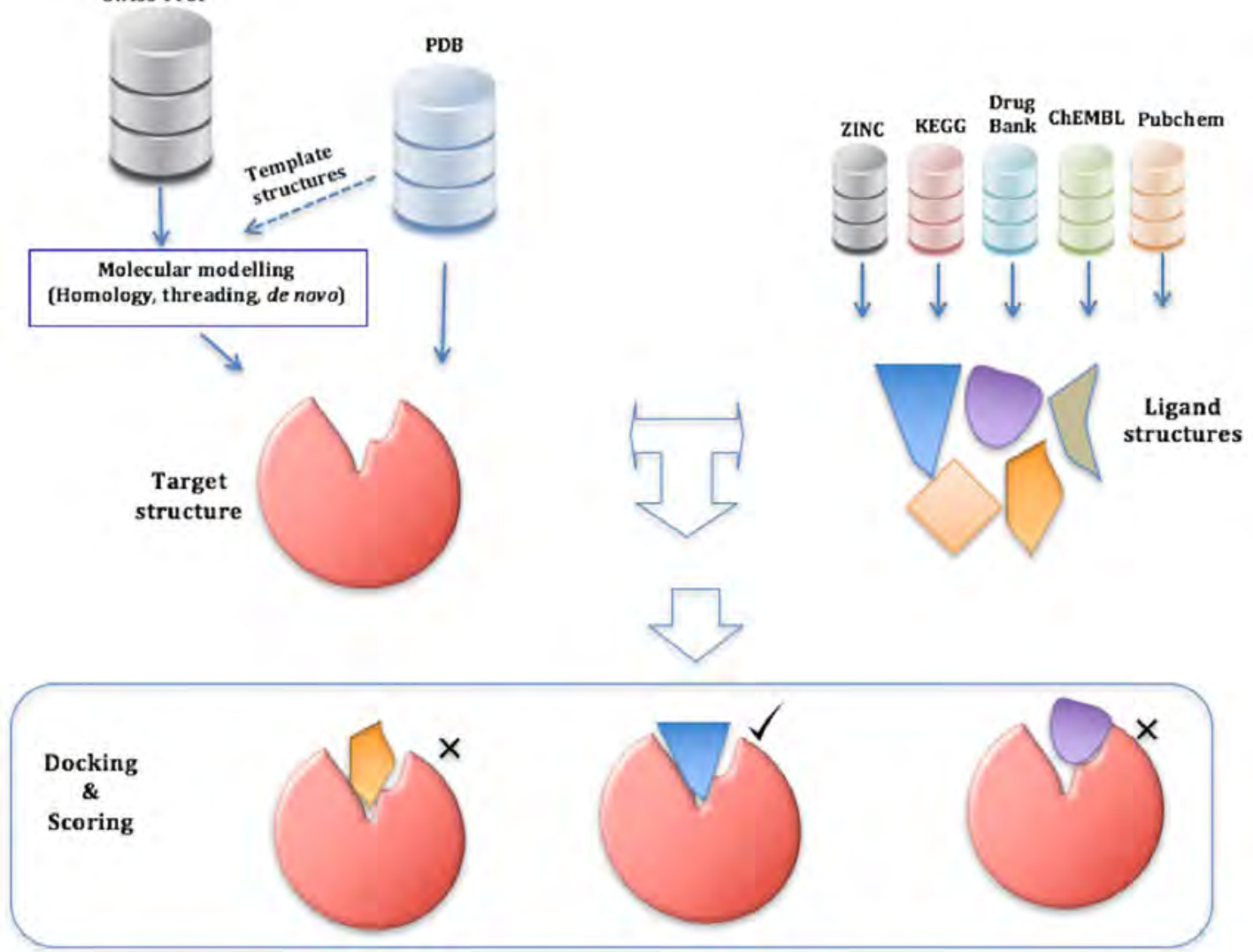

Ligand and Protein as flexible bodies

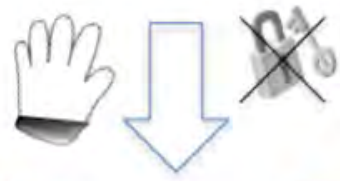

Ligand and Protein as rigid bodies

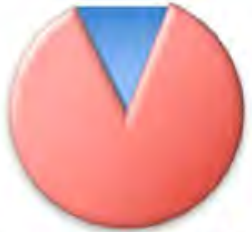

Ideal "best-fit" scenario, where both protein and ligand are flexible.

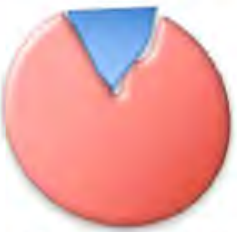

However, current docking protocols mostly account for only ligand flexibility. 

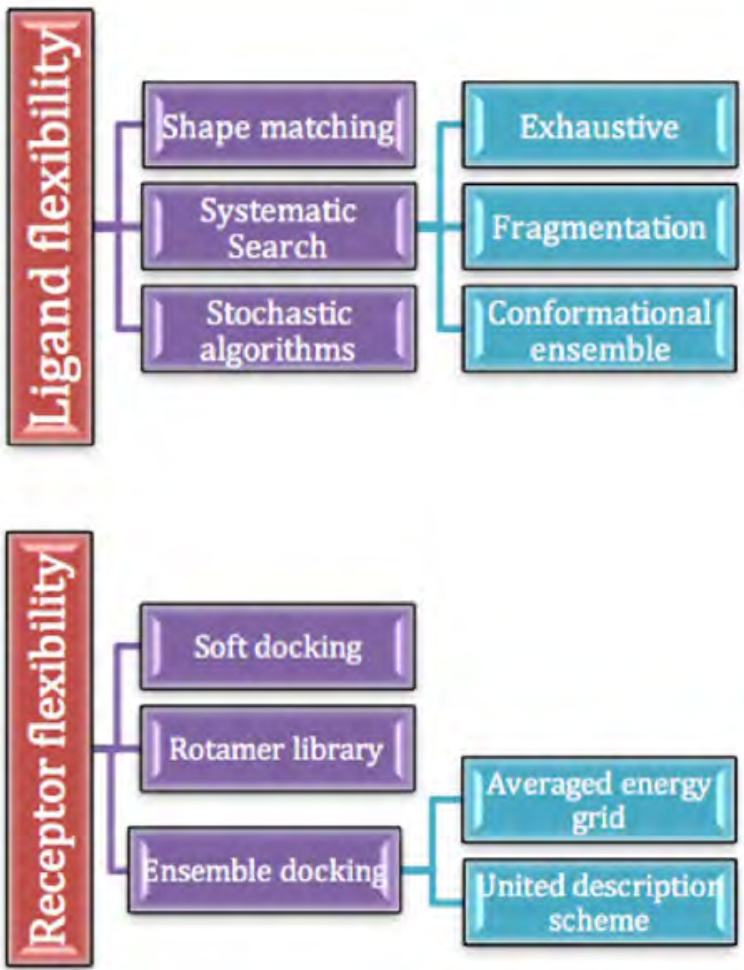

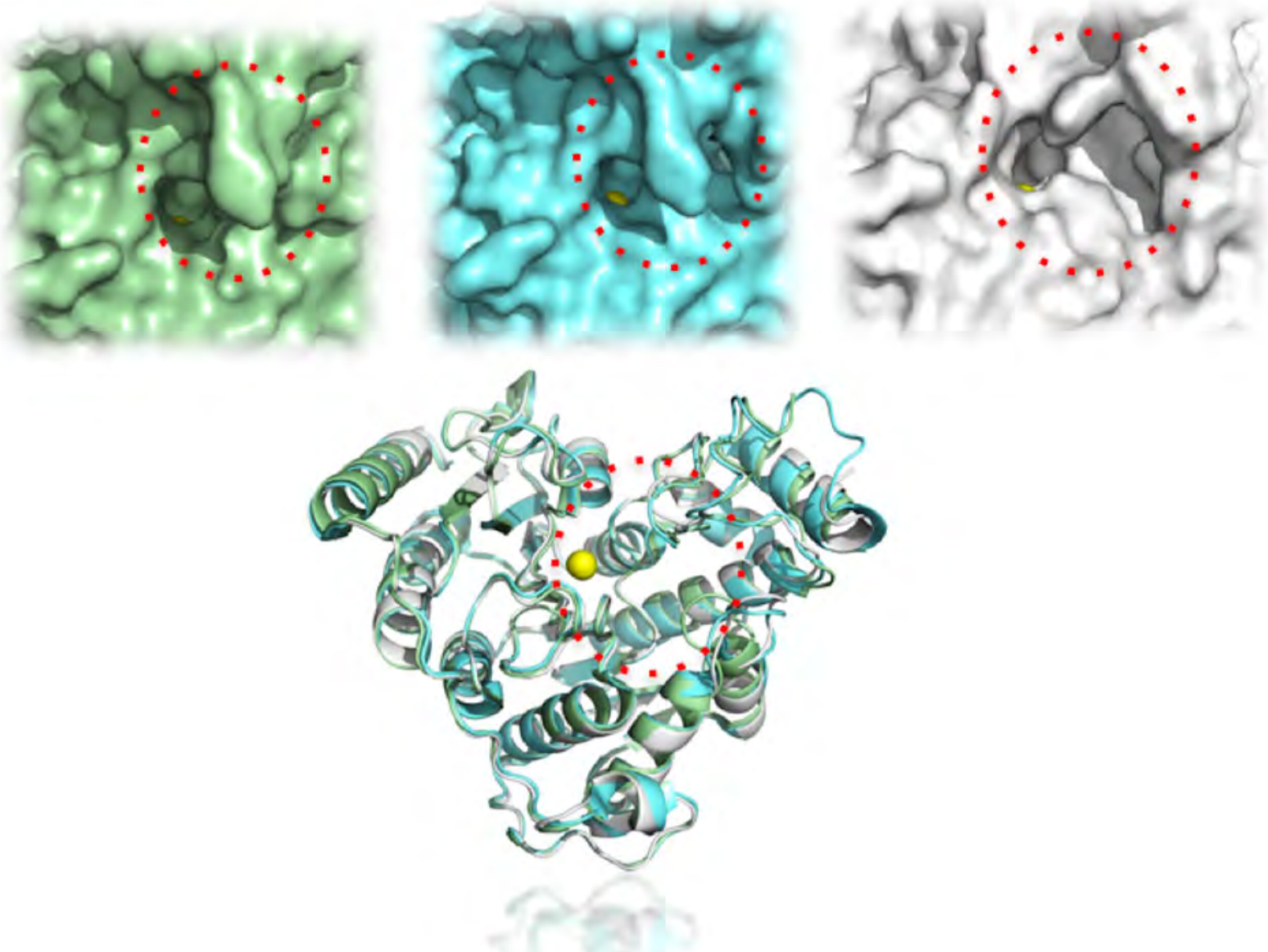


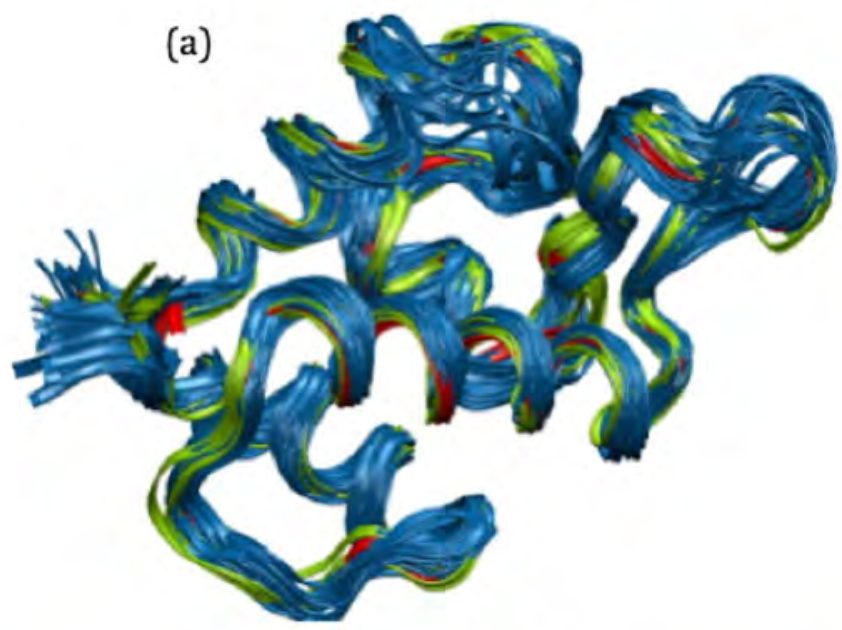

(b)

(c)

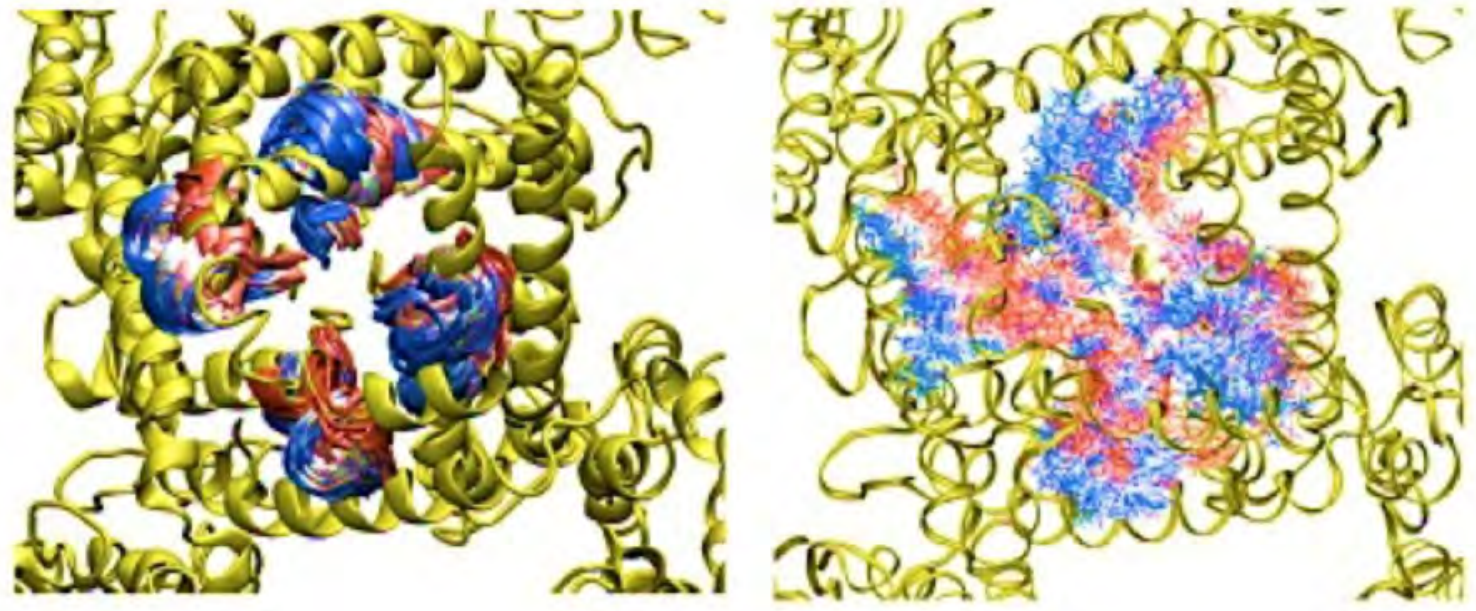




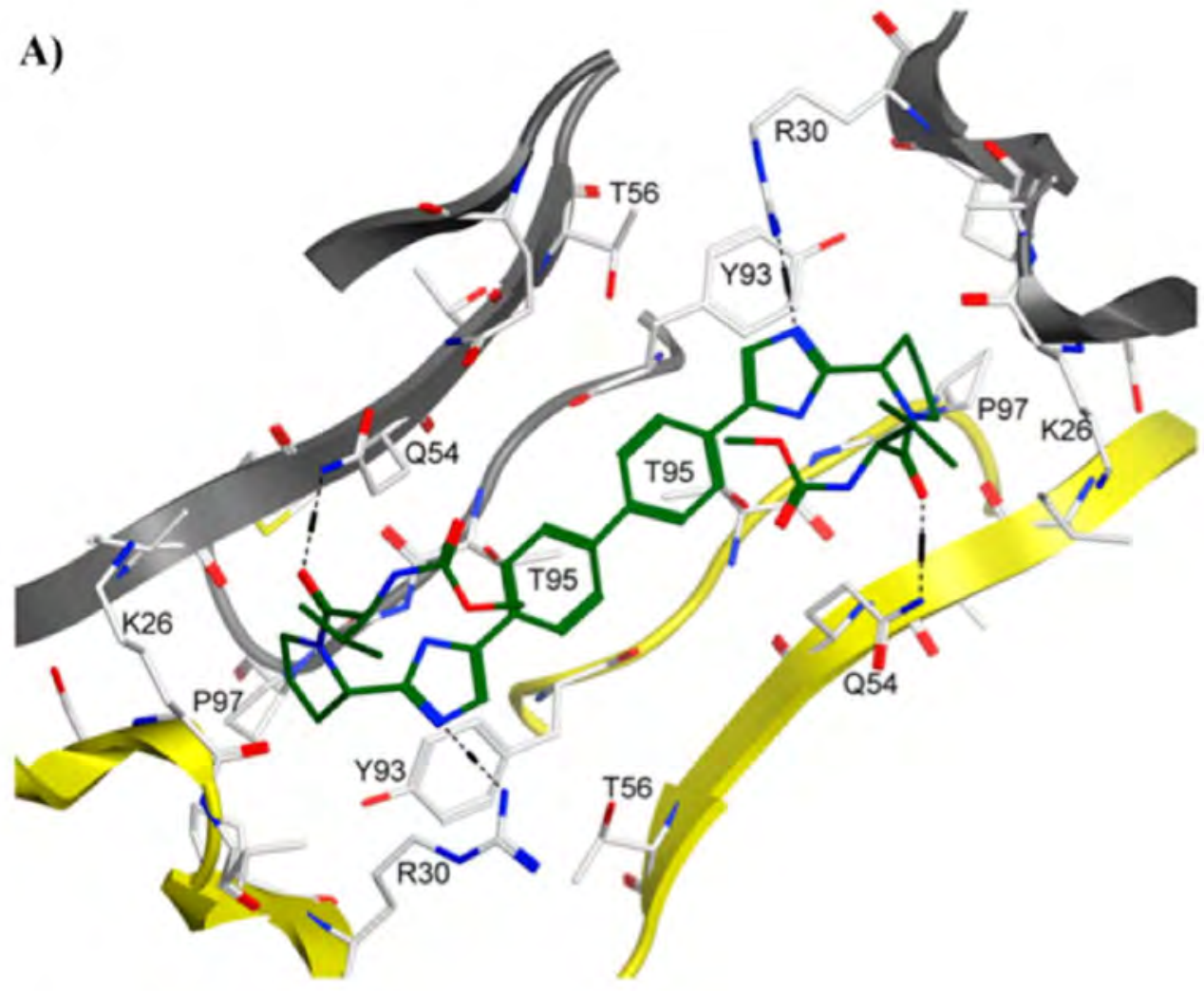

B)

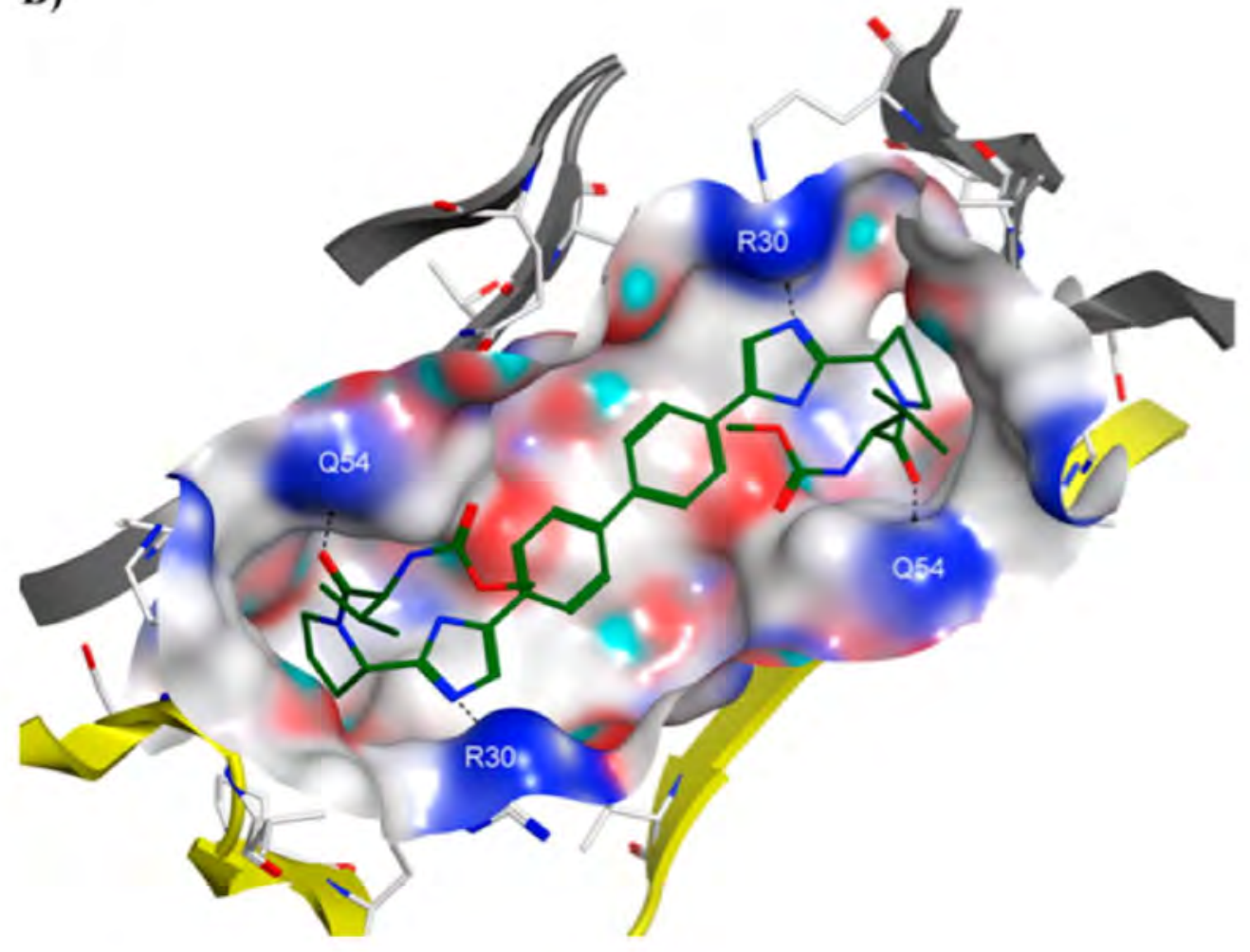




\section{MM}




\section{Prepared initial structure from PDB (or) molecular modelling approach(es)} Important steps \& decision:

(1) If the structure is avallable in the PDB and if yes,

- Whether the structure is complete without any missing residues or segments?

- Whether multople conformations of the target is available in the PDB?

(2) If the structure is not available, then it has to be modelled; and in this case

- What modelling approach(es) and softwaro program(s) are need?

- If fairly good quality tem plate structures are available to model the 30 structure of the target?

- Is the quality of the modelled structure reasonable enough to initiate the next stage?

\section{Classical MD Simulation to gain insights into the structure of the target and to} identify possible druggable pocket(s).

\section{Important steps \& decision:}

(1) Which software program and force-field are suitsble for the MD simulation of the concerned target structures. Does your choice is also supported by the previous work(s) in the literature?

(2) What parameters (such as cell dimension and temperature) are suitable for the system?

(3) What type of water model is appropriate for the system and also compatible with the selected force-field?

(4) How long should the system be equllibrated to obtain a fairly good starting contiguration for the production runs? This decision is always made by verifying the behavior of the system and other physical Quantities, such as temperature, pressure and different energies during the equilibration.

5) What will be the length of production MD simulation and if it is sufficient to sample various conformations of the proteins and also capture the significant dynamics related to ligand binding?

\section{Clustering and selection of dominant conformations to account protein flexibility} and preparation of ensemble of target structures.

Important steps \& decision:

(1) Which clustering algorithm niceds to be used?

(2) Has the trajectory from the preceding MD simulations sampled various dominant conformations that is sufficient for accounting protein flexibility in the further drig design efforts?

\section{Docking based -virtual screening}

Important steps \& decision:

(1) Has the binding site been identified and characterized sufficiently?

(2) Is the grid box suffeiently lange to accommodate the ligands?

(3) How many conformations of Ligands need to be included in screening and how many resultant poses of tigand-target complexes aeed to be collected after screening?

(4) What software and type of scoring functions is required for screening?

(5) Are chemical compound libraries handy and do they indude diverse structural groups?

\section{MD simulation and binding free calculations}

Important steps \& decision:

1) What software program, force-field and parameters are suifable for the MD simulation? These cholces usually remain close to what have been used in MD simulations performed at proviousstages, if any.

(2) How long should the MD simulations need to be fun that could produce fairly accurate binding frec energies? Note, as discussed in this article, there have been several debates on the cholce of one long MD trajectory or multiple short MD trajectorles for this purpose.

(3) What binding free energy method is suitable and also affordable for this reseatch? Guidance from literaturs tan belp in making this choice

\section{Pharmacophore modelling, QSAR} and experimental SAR

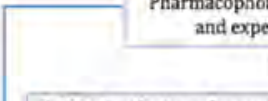

5. MD simulation of promising hits identified from experimental assays

Important steps \& decision

(1) What soltware program, force-field and parameters are sultable for the MO simulation? These choices usually remain dose to what have beeo used in MD simulations performed arprevious stages, if any.

(2) How long should the MD simulations need to be run to refine the post-docking complexes and also to capture the significant dynamic interactions between the target and the bond ligand

6. Employ sophisticated binding free energy methods to identify the best binding mode of the hit. Important steps \& decision:

(1) What binding free energy method is suitable and also affordable for this research? Guidance from literature can help in making this choice.

(2) Can the predicted bioding mode(s) ran be related to the range of activity seen in experiments?

\section{Advanced QM/MM MD simulations to gain deeper Insightsabour the reaction mechanisms involved} between the selected hit(s) and target(s)

Important steps \& decision:

(1) Are the selected ligand-target complexes the best choice to initiate expensive QM/MM MD simulations?

(2) Do previous information about similar interactions and their reaction mechanism avallable in the literature

(3) What would te the optimal seguent of the complex that would require highavevel QM tratment?

(4) Whatis the OM/MM protocol and soltware pregram avallable to perform these simulations?

(5) Is access to high-performance computers availible to carty out these expensive MD simulations?

(5) Is access to high-performance computers available to
(6) What would be the leneth of the MD simulations? 